\title{
Triangular bounded consistency of fuzzy preference relations
}

DOI:

10.1016/j.ins.2018.12.029

\section{Document Version}

Accepted author manuscript

Link to publication record in Manchester Research Explorer

\section{Citation for published version (APA):}

Chang, W., Fu, C., Xu, D., \& Xue, M. (2018). Triangular bounded consistency of fuzzy preference relations. Information Sciences. https://doi.org/10.1016/j.ins.2018.12.029

\section{Published in:}

Information Sciences

\section{Citing this paper}

Please note that where the full-text provided on Manchester Research Explorer is the Author Accepted Manuscript or Proof version this may differ from the final Published version. If citing, it is advised that you check and use the publisher's definitive version.

\section{General rights}

Copyright and moral rights for the publications made accessible in the Research Explorer are retained by the authors and/or other copyright owners and it is a condition of accessing publications that users recognise and abide by the legal requirements associated with these rights.

\section{Takedown policy}

If you believe that this document breaches copyright please refer to the University of Manchester's Takedown Procedures [http://man.ac.uk/04Y6Bo] or contact uml.scholarlycommunications@manchester.ac.uk providing relevant details, so we can investigate your claim.

\section{OPEN ACCESS}




\title{
Triangular bounded consistency of fuzzy preference relations
}

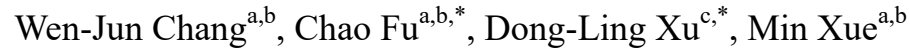 \\ ${ }^{a}$ School of Management, Hefei University of Technology, Hefei, Box 270, Hefei 230009, Anhui, P.R. China \\ ${ }^{\mathrm{b}}$ Key Laboratory of Process Optimization and Intelligent Decision-making, Ministry of Education, Hefei 230009 ,
}

Anhui, P.R. China

${ }^{\mathrm{c} D e c i s i o n}$ and Cognitive Sciences Research Centre, Alliance Manchester Business School, The University of

Manchester, Manchester M13 9SS, UK

\begin{abstract}
There are typically two types of consistency of fuzzy preference relations (FPR), namely additive and multiplicative consistency. They are defined based on the assumption that decision makers are rational and can provide strictly consistent FPRs. To take into consideration the bounded rationality of decision makers, the current study relaxes this assumption and proposes a new measure called triangular bounded consistency. To define triangular bounded consistency, a directed triangle is used to represent three FPRs among any three alternatives, with each directed edge representing an FPR. The condition of restricted max-max transitivity (RMMT) in the directed triangle is quantitatively examined. Under the assumption that the bounded rationality of a decision maker is characterized by their historical FPRs, which are represented by directed triangles that satisfy RMMT, triangular bounded consistency is determined using the historical FPRs. We then illustrate how triangular bounded consistency can be used to verify the consistency of FPRs that are newly provided by decision makers and how to estimate some missing FPRs that are not provided by decision makers. Finally, to demonstrate the application of triangular bounded consistency of FPRs in multi-attribute decision analysis, we investigate a problem that involves selecting areas to market products for a company.
\end{abstract}

Keywords: Triangular bounded consistency; Fuzzy preference relation; Restricted max-max transitivity; Bounded rationality; Multi-attribute decision analysis

\footnotetext{
${ }^{*}$ Corresponding author. Tel: 0086551 62904930, 0044161 3063427; fax: 008655162905263 ,
} 
00441613063505.

E-mail address:wls_fuchao@163.com(C.Fu),1.xu@manchester.ac.uk (D.-L. Xu).

\section{Introduction}

Decision makers generally use preference relations to express their preference information when analyzing decision problems (Herrera et al., 2001; Massanet et al., 2016; Wan et al., 2018). The two types of preference relations commonly accepted in the context of pairwise comparison of alternative courses of action (or simply, alternatives) (Genç et al., 2010; Chen et al., 2015; Wan et al., 2017) are multiplicative preference relation (MPR) (Saaty, 1977) and fuzzy preference relation (FPR) (Orlovsky, 1978). As the two types of preference relations can be transformed into each other (Herrera-Viedma et al., 2004), this study focuses on FPR. FPR was first proposed by Orlovsky (1978) to represent the opinion of a decision maker when comparing a set of alternatives. FPR can not only reflect whether an alternative is superior to another but also characterize the degree to which an alternative is preferred (Pan et al., 2017; Zhang et al., 2018). The full definition of FPR is given in Section 2.

Consistency is a prerequisite for using FPRs to model and analyze decision problems (Chen et al., 2014; Li et al., 2018). Verifying whether the required consistency is reached is an important step in the process of analyzing decision problems modeled by FPRs (Ureña et al., 2015; Zhang et al., 2016). Decisions made using inconsistent FPRs may be irrational and of poor quality (Xu et al., 2016; Herowati et al., 2017). A basic requirement for consistency is transitivity. Different types of transitivity, such as weak transitivity, triangular condition, and restricted max-max transitivity (RMMT), have been defined and investigated in existing studies (Liao and Xu, 2015; Liu et al., 2018). RMMT is stricter than weak transitivity and triangular condition (see Section 2) and is 
considered as a necessary condition of consistent FPRs (Liu et al., 2014).

To verify the consistency of FPRs, different types of consistency measure of FPR have been developed (Chiclana et al., 2009; Deng et al., 2014; Deng et al., 2015). Representative and commonly accepted types include additive consistency (Pérez et al., 2016; Al Salema and Awasthi, 2018) and multiplicative consistency (Wu and Chiclana, 2014; Krejčí, 2017). Both of these types of consistency satisfy RMMT (Herrera-Viedma et al., 2004). More importantly, they are based on the assumption that decision makers are strictly rational, and thus the FPRs strictly satisfy the given mathematical conditions for the two types of consistency (Herrera-Viedma et al., 2004; Xu et al., 2014). However, it is very difficult or even impossible in practice for decision makers to offer such exact FPRs as they may not have access to all the information required; even if they do, they may not be able to process it properly within a given time limit (Wang and Chen, 2008; Yan and Ma, 2015). This phenomenon is referred to as bounded rationality by Simon (1982). Therefore, in many situations, decision makers may seek to offer "good enough" judgments that may not be perfectly rational or consistent, but reasonably acceptable. Hence, we must consider which judgements are reasonably acceptable and can be used for decision-making.

Therefore, we propose a new consistency concept called triangular bounded consistency to measure the consistency of FPRs. Firstly, the difference between the two commonly accepted types of consistency of FPR, i.e., additive and multiplicative consistency, and then their limitations are analyzed. Secondly, inspired by triangular condition (Dasgupta and Deb, 1996), which is a type of transitivity of FPR, we propose the use of a directed triangle to represent the three FPRs among any three alternatives in which each FPR is represented by a directed edge. Because RMMT is considered as a necessary condition for consistent FPRs, we then examine what the 
triangles should look like if the FPRs they represent satisfy RMMT by specifying the range of angles formed by two adjacent edges. Here, adjacent edges represent the two directed edges whose arrows do not both leave or point to the angles they form. That is, the arrow of one directed edge should point to the angle, whereas the arrow of the other edge should point away from the angle. When the collected preference information satisfies RMMT, it is regarded as reasonably acceptable. Otherwise, it should be further revised or ignored. In other words, we assume that all decision makers are at least reasonably rational in the sense that their preferences satisfy RMMT. Thirdly, we introduce the concept of triangular bounded consistency to measure the consistency of FPRs provided by a decision maker. To estimate triangular bounded consistency of given FPRs, the pairwise preference judgments provided by the decision maker previously, or currently to a set of sample alternatives if no previous judgments are available, should be collected. From the collected information, the preferences satisfying the RMMT can be represented by directed triangles and then used to estimate the lower and upper bounds of triangular bounded consistency of FPRs for this particular decision maker.

One important use of triangular bounded consistency is estimating missing FPRs, as decision makers generally offer only minimal preference information (Sen and Yang, 1994) or an acceptable incomplete FPR matrix (which is defined in Section 2) to avoid inconsistency issues when there are many alternatives under consideration. For example, among alternatives $i, j$, and $k$, a decision maker may specify how much they prefer $i$ to $j$ and $j$ to $k$ without mentioning their preference between $i$ and $k$. When this is the case, we can estimate their preferences between $i$ and $k$ using triangular bounded consistency estimated for them. As the estimated preference is normally a range, to find a point estimate of the missing FPR, an optimization model is 
constructed using the least squares method to minimize the difference between the point estimate and the decision maker's usual or historical consistency behavior characterized by the middle point of the range of their triangular bounded consistency.

To demonstrate the application of triangular bounded consistency, we applied this concept to analyze multi-attribute decision analysis (MADA) problems modeled by acceptable incomplete FPR matrices. The main contributions of this study can be summarized as follows: (1) we analyze the difference between additive and multiplicative consistency of FPRs, (2) we specify the value range of the angle formed by two adjacent edges of the directed triangle representing FPRs that satisfy RMMT, (3) we introduce a new consistency concept called triangular bounded consistency to verify the consistency of newly provided FPRs by a decision maker to follow their usual consistency behavior, (4) we apply triangular bounded consistency to find the missing FPRs in an incomplete FPR matrix provided by a decision maker, and (5) we develop a solution process to MADA problems modeled by acceptable incomplete FPR matrices.

The remainder of this paper is organized as follows. Section 2 presents the basics of our proposed consistency concept. Section 3 provides an analysis of additive and multiplicative consistency of FPRs. Section 4 introduces triangular bounded consistency and uses it to estimate missing elements in acceptable incomplete FPR matrices. Section 5 describes the application of triangular bounded consistency to MADA problems modeled by FPRs. This application is demonstrated in Section 6 by analyzing a problem of selecting areas to market the products of a company. Finally, Section 7 presents conclusions and future work to be considered.

\section{Preliminaries}


In this section, we introduce the basic concepts of FPRs, some representative definitions of consistency and transitivity of FPRs, and the validity judgment on an incomplete FPR matrix with missing elements.

Definition 1. (Orlovsky, 1978) Let $X=\left\{x_{1}, x_{2}, \ldots, x_{n}\right\}$ be a set of alternatives. $P=\left(p_{i j}\right)_{n \times n}$ is called an FPR matrix on $X \times X$ such that $p_{i j} \in[0,1], p_{i j}+p_{j i}=1, \forall i, j \in\{1, \ldots, n\}$, where $p_{i j}$ denotes the degree to which alternative $x_{i}$ is preferred to $x_{j}$. Specifically, $p_{i j}=0.5$ indicates an indifference between $x_{i}$ and $x_{j}$ (denoted as $x_{i}: x_{j}$ ), $0.5<p_{i j}<1$ indicates that $x_{i}$ is preferred to $x_{j}$ (denoted as $x_{i} \mathrm{f} x_{j}$ ), and $p_{i j}=1$ indicates that $x_{i}$ is absolutely preferred to $x_{j}$.

As mentioned in Section 1, consistency is an important property with respect to FPRs. In previous studies, the two commonly accepted definitions of consistency, namely additive and multiplicative consistency, were used to determine whether an FPR matrix is consistent.

Definition 2. (Tanino, 1984) Given a set of alternatives $X=\left\{x_{1}, x_{2}, \ldots, x_{n}\right\}$, let $P=\left(p_{i j}\right)_{n \times n}$ be an FPR matrix on $X \times X$. $P$ is considered additively consistent if it satisfies the following condition:

$$
p_{i j}+p_{j k}+p_{k i}=1.5, \quad \forall i, j, k \in\{1, \ldots, n\} .
$$

Definition 3. (Tanino, 1984) Given a set of alternatives $X=\left\{x_{1}, x_{2}, \ldots, x_{n}\right\}$, let $P=\left(p_{i j}\right)_{n \times n}$ be an FPR matrix on $X \times X . P$ is considered multiplicatively consistent if it satisfies the following condition:

$$
p_{i j} \cdot p_{j k} \cdot p_{k i}=p_{i k} \cdot p_{k j} \cdot p_{j i}, \quad \forall i, j, k \in\{1, \ldots, n\} .
$$

From the above two definitions, it can be observed that additive and multiplicative consistency are very strict conditions for FPRs to satisfy. Compared with the two types of consistency, transitivity is a weaker condition for FPRs to satisfy. Representative types of transitivity include weak transitivity, RMMT, and triangular condition. 
Definition 4. (Tanino, 1984; Herrera-Viedma et al., 2004) Given a set of alternatives $X=\left\{x_{1}\right.$, $\left.x_{2}, \ldots, x_{n}\right\}$, let $P=\left(p_{i j}\right)_{n \times n}$ be an FPR matrix on $X \times X . P$ is said to be weakly transitive if it satisfies the following conditions:

(1) if $0.5 \leq p_{i j} \leq 1$ and $0.5 \leq p_{j k} \leq 1$, then $0.5 \leq p_{i k} \leq 1, \forall i, j, k \in\{1, \ldots, n\}$, and

(2) if $0 \leq p_{i j} \leq 0.5$ and $0 \leq p_{j k} \leq 0.5$, then $0 \leq p_{i k} \leq 0.5, \forall i, j, k \in\{1, \ldots, n\}$.

Definition 5. (Herrera-Viedma et al., 2004) Given a set of alternatives $X=\left\{x_{1}, x_{2}, \ldots, x_{n}\right\}$, let $P=$ $\left(p_{i j}\right)_{n \times n}$ be an FPR matrix on $X \times X . P$ is said to have RMMT if it satisfies the following conditions:

(1) if $0.5 \leq p_{i j} \leq 1$ and $0.5 \leq p_{j k} \leq 1$, then $\max \left\{p_{i j}, p_{j k}\right\} \leq p_{i k} \leq 1, \forall i, j, k \in\{1, \ldots, n\}$, and

(2) if $0 \leq p_{i j} \leq 0.5$ and $0 \leq p_{j k} \leq 0.5$, then $0 \leq p_{i k} \leq \min \left\{p_{i j}, p_{j k}\right\}, \forall i, j, k \in\{1, \ldots, n\}$.

Definition 6. (Dasgupta and Deb, 1996; Herrera-Viedma et al., 2004) Given a set of alternatives $X$ $=\left\{x_{1}, x_{2}, \ldots, x_{n}\right\}$, let $P=\left(p_{i j}\right)_{n \times n}$ be an FPR matrix on $X \times X . P$ is said to satisfy triangular condition if

$p_{i j}+p_{j k} \geq p_{i k}, \quad \forall i, j, k \in\{1, \ldots, n\}$.

RMMT is stricter than weak transitivity and triangular condition, and it is generally regarded as a necessary condition for a consistent FPR matrix. An FPR matrix satisfying additive or multiplicative consistency implies that RMMT is satisfied in the matrix, which is formally described in the following theorems.

Theorem 1. (Herrera-Viedma et al., 2004) Given a set of alternatives $X=\left\{x_{1}, x_{2}, \ldots, x_{n}\right\}$, let $P=$ $\left(p_{i j}\right)_{n \times n}$ be an FPR matrix on $X \times X$. If $P$ satisfies multiplicative consistency, i.e., if it satisfies $p_{i j} \cdot p_{j k} \cdot p_{k i}=p_{i k} \cdot p_{k j} \cdot p_{j i}, \quad \forall i, j, k \in\{1, \ldots, n\}, i \neq j \neq k$, then $P$ must satisfy RMMT.

Theorem 2. (Herrera-Viedma et al., 2004; Liao and Xu, 2014) Given a set of alternatives $X=\left\{x_{1}\right.$, $\left.x_{2}, \ldots, x_{n}\right\}$, let $P=\left(p_{i j}\right)_{n \times n}$ be an FPR matrix on $X \times X$. If $P$ satisfies additive consistency, i.e., if it 
satisfies $p_{i j}+p_{j k}+p_{i k}=1.5, \forall i, j, k \in\{1, \ldots, n\}, i \neq j \neq k$, then $P$ must satisfy RMMT.

When some elements in an FPR matrix $P$ cannot be provided by a decision maker, $P$ is called an incomplete FPR matrix.

Definition 7. (Ureña et al., 2015) Given a set of alternatives $X=\left\{x_{1}, x_{2}, \ldots, x_{n}\right\}$, let $P=\left(p_{i j}\right)_{n \times n}$ be an FPR matrix on $X \times X$. If some elements other than the diagonal ones in $P$ cannot be provided, then $P$ is called an incomplete FPR matrix, which satisfies

$$
p_{i j} \in[0,1], p_{i j}+p_{j i}=1, \quad \forall i, j \in\{1, \ldots, n\} .
$$

Next, we discuss whether an incomplete FPR matrix is acceptable or not.

Definition 8. (Xu et al., 2014) Given a set of alternatives $X=\left\{x_{1}, x_{2}, \ldots, x_{n}\right\}$, let $P=\left(p_{i j}\right)_{n \times n}$ be an incomplete FPR matrix on $X \times X$. If the missing elements of $P$ can be determined by its provided elements, then $P$ is called an acceptable incomplete FPR matrix; otherwise, $P$ is not an acceptable incomplete FPR matrix.

Theorem 3. (Xu et al., 2014) Given a set of alternatives $X=\left\{x_{1}, x_{2}, \ldots, x_{n}\right\}$, let $P=\left(p_{i j}\right)_{n \times n}$ be an incomplete FPR matrix on $X \times X$. Only when there exists at least one provided element in each row or column of $P$, with exception of the diagonal elements $\left(p_{i i}=0.5, i=1, \ldots, n\right)$, the matrix can be considered acceptable.

\section{Analysis of existing consistency of FPRs}

Section 2 presents the two commonly accepted types of consistency of FPRs, i.e., additive and multiplicative consistency. In the following, they are analyzed to show their difference and possible limitations in real applications.

Given an MPR matrix $A=\left(a_{i j}\right)_{n \times n}$ on $X \times X$ with $a_{i j} \in[1 / 9,9]$, where $X=\left\{x_{1}, x_{2}, \ldots, x_{n}\right\}$ denotes a set of alternatives, it is said to be consistent if we have $a_{i j} \cdot a_{j k}=a_{i k}, \forall i, j, k$. Owing to the 
transformation of an MPR matrix to an FPR matrix, which is demonstrated by Proposition B.1 of Section B.1 in Appendix B of the supplementary material, a consistent MPR matrix can result in a consistent FPR matrix satisfying the condition in Definition 2 presented in Section 2. It can be inferred from the equivalence between the consistency of an MPR matrix and the additive consistency of an FPR matrix that a decision maker is said to be perfectly rational when they provide a consistent FPR matrix. However, multiplicative consistency of an FPR matrix cannot reflect the perfect rationality of a decision maker because a difference exists between additive and multiplicative consistency of an FPR matrix. To facilitate a quantitative measurement of the deviation between additive and multiplicative consistency, the following proposition is given first.

Proposition 1. Suppose that $f(x, y)=\frac{x y}{1-x-y+2 x y}-(x+y-0.5)$ is a two-variable function with $0 \leq x, y \leq 1$. Then, the function satisfies

(1) when $x, y \in[0.5,1], f(x, y)$ is a monotonously decreasing function with respect to $x$ or $y$.

(2) when $x, y \in[0,0.5], f(x, y)$ is a monotonously decreasing function with respect to $x$ or $y$.

(3) when $(x-0.5) \cdot(y-0.5) \leq 0$, the lower bound of $f(x, y)$ tends to -0.5 if $(x, y)=\left(0,1^{-}\right)$or $(x, y)$ $=\left(1^{-}, 0\right)$ while the upper bound of $f(x, y)$ tends to 0.5 if $(x, y)=\left(0^{+}, 1\right)$ or $(x, y)=\left(1,0^{+}\right)$.

(4) when $(x-0.5) \cdot(y-0.5) \geq 0, f(x, y)$ reaches the maximum value, i.e., -0.5 , if $(x, y)=(1,1)$, while $f(x, y)$ reaches the minimum value, i.e., 0.5 , if $(x, y)=(0,0)$.

The proof of Proposition 1 is presented in Section A.1 of Appendix A in the supplementary material. It can be deduced from Definitions 2 and 3 presented in Section 2 that $p_{i k}=p_{i j}+p_{j k}-0.5$ $(\forall i, j, k \in\{1, \ldots, n\})$ holds in an additively consistent FPR matrix, and $p_{i k}=$ $\frac{p_{i j} p_{j k}}{1-p_{i j}-p_{j k}+2 p_{i j} p_{j k}}(\forall i, j, k \in\{1, \ldots, n\})$ holds in a multiplicatively consistent FPR matrix. Assume that $p_{i k}^{a}=p_{i j}+p_{j k}-0.5(\forall i, j, k \in\{1, \ldots, n\})$ is an additively consistent FPR matrix, 
and $p_{i k}^{m}=\frac{p_{i j} p_{j k}}{1-p_{i j}-p_{j k}+2 p_{i j} p_{j k}}(\forall i, j, k \in\{1, \ldots, n\})$ is a multiplicatively consistent FPR matrix. Then, the function $f(x, y)$ in Proposition 1 can represent the deviation between $p_{i k}^{m}$ and $p_{i k}^{a}$ given that $p_{i j}$ and $p_{j k}$ are two variables. Proposition 1 also reveals that $-0.5 \leq p_{i k}^{m}-p_{i k}^{a} \leq 0.5$ when $\left(p_{i j}-0.5\right) \cdot\left(p_{j k}-0.5\right) \geq 0$, and $-0.5<p_{i k}^{m}-p_{i k}^{a}<0.5$ when $\left(p_{i j}-0.5\right) \cdot\left(p_{j k}-0.5\right) \leq 0$. It can be easily concluded that multiplicative consistency of an FPR matrix is different from additive consistency of the matrix, particularly when $\left(p_{i j}-0.5\right) \cdot\left(p_{j k}-0.5\right) \leq 0(\forall i, j, k \in\{1, \ldots, n\})$. As such, multiplicative consistency is incapable of characterizing the perfect rationality of a decision maker.

Example 1. For a set of alternatives $X=\left\{x_{1}, \ldots, x_{5}\right\}$, suppose that $P=\left(p_{i j}\right)_{5 \times 5}$ is an FPR matrix on $X \times X$ with $p_{12}=0.7, p_{23}=0.6, p_{34}=0.98$, and $p_{45}=0.1$. The deviations between $p_{13}^{m}$ and $p_{13}^{a}$ and between $p_{35}^{m}$ and $p_{35}^{a}$ are calculated in the following.

As for $p_{12}$ and $p_{23}$, we have $p_{13}^{a}=p_{12}+p_{23}-0.5=0.8$ and $p_{13}^{m}=\frac{p_{12} p_{23}}{1-p_{12}-p_{23}+2 p_{12} p_{23}}=$ 0.78 ; therefore, the deviation between $p_{13}^{m}$ and $p_{13}^{a}$ is -0.02 . When $p_{34}$ and $p_{45}$ are used to consistently decide $p_{35}$, it can be similarly calculated that $p_{35}^{a}=p_{34}+p_{45}-0.5=0.58$ and $p_{35}^{m}=$ $\frac{p_{34} p_{45}}{1-p_{34}-p_{45}+2 p_{34} p_{45}}=0.84$, which implies that $p_{35}^{m}-p_{35}^{a}=0.26$. The results in these two situations are clearly limited to the lower and upper bounds given in Proposition 1.

Specifically, $p_{12}=0$ and $p_{23}=0.999$ lead to $p_{13}^{m}-p_{13}^{a}=-0.499$, and $p_{34}=0.001$ and $p_{45}=1$ result in $p_{35}^{m}-p_{35}^{a}=0.499$. This verifies Proposition 1 under the condition that $\left(p_{i j}-0.5\right) \cdot\left(p_{j k}-0.5\right)$ $\leq 0$. As a whole, a clear difference can be noted between multiplicative and additive consistency of an FPR matrix from perfect rationality.

In addition, another important difference is noted between multiplicative and additive consistency of an FPR matrix. That is, given $p_{i j}$ and $p_{j k}$, the domain of $p_{i k}$ for multiplicative 
consistency is different from the domain of $p_{i k}$ for additive consistency. To facilitate such comparison, the following proposition is given first.

Proposition 2. Suppose that $f(x, y)=\frac{x y}{1-x-y+2 x y}$ is a two-variable function with $0 \leq x, y \leq 1$. Then, the function is monotonously increasing with respect to $x$ or $y$.

The proof of Proposition 2 is presented in Section A.2 of Appendix A. This proposition indicates that $p_{i k}$ generated by multiplicative consistency is always limited to the interval $[0,1]$ given $p_{i j}$ and $p_{j k}$ such that $0 \leq p_{i j}, p_{j k} \leq 1$. In detail, it can be determined from Proposition 2 that $p_{i k}$ $=0$ when $\left(p_{i j}, p_{j k}\right)=(0,0)$, and $p_{i k}=1$ when $\left(p_{i j}, p_{j k}\right)=(1,1)$ under the condition that multiplicative consistency is satisfied. On the other hand, when $p_{i j}$ and $p_{j k}$ such that $0 \leq p_{i j}, p_{j k} \leq 1$ are given to determine $p_{i k}$ using additive consistency, the range of $p_{i k}$ is limited to the interval $[-1.5,1.5]$. When $\left(p_{i j}, p_{j k}\right)=(0,0), p_{i k}$ is equal to -1.5 , whereas $p_{i k}$ becomes 1.5 when $\left(p_{i j}, p_{j k}\right)=(1,1)$. As a result, the range of $p_{i k}$ from additive consistency is clearly different from the range of multiplicative consistency given $p_{i j}$ and $p_{j k}$ such that $0 \leq p_{i j}, p_{j k} \leq 1$.

Although the range of $p_{i k}$ from additive consistency given $p_{i j}$ and $p_{j k}$ such that $0 \leq p_{i j}, p_{j k} \leq 1$ can be mapped into the interval $[0,1]$ in some way (Herrera-Viedma et al., 2004), the decision maker providing the FPRs is assumed to be perfectly rational. Meanwhile, the assumption of perfect rationality is relaxed when multiplicative consistency is applied. In real applications, it is very difficult or even impossible for a decision maker to be perfectly rational and provide an additively consistent FPR matrix. More importantly, both additive and multiplicative consistency cannot reflect the real preference of a decision maker with bounded rationality. In other words, for any pair of $p_{i j}$ and $p_{j k}, p_{i k}$ can be generated directly by additive or multiplicative consistency without consideration of the preference of a decision maker. As such, the resulting $p_{i k}$ cannot be 
guaranteed to be the one that the decision maker wishes to provide. In the context of decision-making, the direct application of additive and multiplicative consistency is doubtful, owing to a lack of consideration of the preferences of a decision maker with bounded rationality.

For these reasons, we develop a new consistency measure of an FPR matrix based on triangular condition in consideration of the preference information of a decision maker with bounded rationality, as described in the next section.

\section{Triangular bounded consistency}

In this section, starting from triangular condition in Definition 6 presented in Section 2, we quantitatively explain the relationship among three edges of a valid triangle and the RMMT in a triangular context. Then, we propose triangular bounded consistency of an FPR matrix with coverage of the preferences of a decision maker.

\subsection{Quantitative description of triangular condition}

In Definition 6, the basic condition of a triangle is used to geometrically explain the consistency condition of an FPR matrix. In the following, we quantitatively describe the relationship among three edges of a directed triangle with the angle between two edges.

From an abstract angle, suppose that $x, y$, and $z$ with $0 \leq x, y, z \leq 2$ denote the three edges of a directed triangle $\triangle A B C$, i.e., edges $\stackrel{\text { umut }}{A B}, \stackrel{\text { umu }}{B C}$, and $\stackrel{\text { um }}{C A}$ in Fig. 1 . The angle between $x$ and $y$ is denoted by $\theta$.

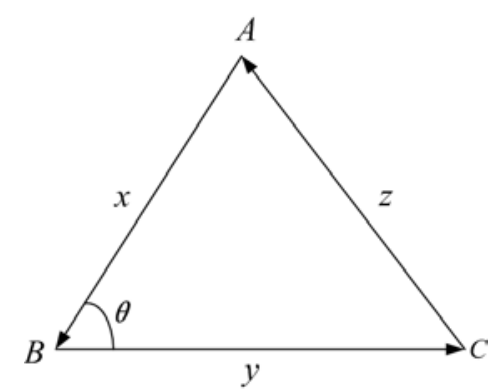


Fig. 1. Directed triangle $\triangle A B C$.

When $x, y$, and $z$ form a triangle, they must satisfy the following conditions:

(1) $x+y \geq z$,

(2) $x+z \geq y$, and

(3) $y+z \geq x$

From the above conditions, we can deduce that $|x-y| \leq z \leq x+y$. The angle between $x$ and $y$ can be constructed from $x, y$, and $z$ as $\theta=\arccos \frac{x^{2}+y^{2}-z^{2}}{2 x y}$. The relationship between $\theta$ and $z$ and the relationship between $\theta$ and $x$ (or $y$ ) are revealed in the following.

Property 1. Suppose that $x, y$, and $z$ with $0 \leq x, y, z \leq 2$ are the lengths of three edges of the triangle $\triangle A B C$ plotted in Fig. 1 with the angle between edges $\stackrel{\text { uut }}{A B}$ and $\stackrel{\text { uu }}{B C}$ denoted by $\theta$. Then, we have

(1) $\theta$ is monotonously increasing with respect to $z$, and

(2) $\theta$ is monotonously decreasing with respect to $x$ or $y$ when $z \geq \sqrt{\left|x^{2}-y^{2}\right|}$.

The proof of Property 1 is presented in Section A.3 of Appendix A. Given $x$ and $y$ such that $0 \leq$ $x, y \leq 2$ and $x>y$, it can be determined that $x-y \leq z \leq x+y$. In this situation, we can deduce from Property 1 that $0 \leq \theta \leq \pi$. This is formally presented in the following.

Property 2. Suppose that $x, y$, and $z$, such that $0 \leq x, y, z \leq 2$ and $x>y$, are the lengths of three edges of the triangle $\triangle A B C$ plotted in Fig. 1 with the angle between edges $\stackrel{\text { umu }}{A B}$ and $\underset{B C}{\text { um }}$ denoted by $\theta$. Then, we have

(1) $0 \leq \theta \leq \pi$,

(2) $\theta=0$ when $z=x-y$, and

(3) $\theta=\pi$ when $z=x+y$. 


\subsection{Description of RMMT in directed triangles of three FPRs}

As demonstrated in Section 2, although RMMT is covered by additive and multiplicative consistency, it is stricter than weak transitivity and triangular condition. In the developed triangular bounded consistency of an FPR matrix, it is also intended to satisfy RMMT.

Given an FPR matrix $P=\left(p_{i j}\right)_{n \times n}$ on $X \times X$, where $X=\left\{x_{1}, x_{2}, \ldots, x_{n}\right\}$ denotes a set of alternatives, assume that $x_{i}, x_{j}$, and $x_{k}$ are the three vertices of a triangle, and FPRs $p_{i j}, p_{j k}$, and $p_{i k}$ represent the lengths of directed edges from vertexes $x_{i}$ to $x_{j}, x_{j}$ to $x_{k}$, and $x_{i}$ to $x_{k}$, respectively. The directed edge from vertex $x_{i}$ to $x_{j}$ means that alternative $x_{i}$ is superior or equivalent to $x_{j}$, which results in $p_{i j} \geq 0.5$. In general, there are two situations where $\left(p_{i j}-0.5\right) \cdot\left(p_{j k}-0.5\right) \geq 0$ and $\left(p_{i j}-\right.$ $0.5) \cdot\left(p_{j k}-0.5\right) \leq 0$, as plotted in Fig. 2. In the first situation, alternative $x_{i}$ is superior or equivalent to $x_{j}$, and $x_{j}$ is superior or equivalent to $x_{k}$; thus, $x_{i}$ is certainly superior or equivalent to $x_{k}$. In other words, $p_{i j} \geq 0.5$ and $p_{j k} \geq 0.5$ must lead to $p_{i k} \geq 0.5$. In the second situation, alternative $x_{i}$ is superior or equivalent to $x_{j}$, and $x_{j}$ is inferior or equivalent to $x_{k}$; whether $x_{i}$ is superior or equivalent to $x_{k}$ depends on whether $p_{i j}$ is larger than or equal to $p_{k j}$ (i.e., $1-p_{j k}$ ). Situation $(b)$ in Fig. 2 indicates that $p_{i j} \geq p_{k j}$. In the following, we discuss RMMT in these two situations.

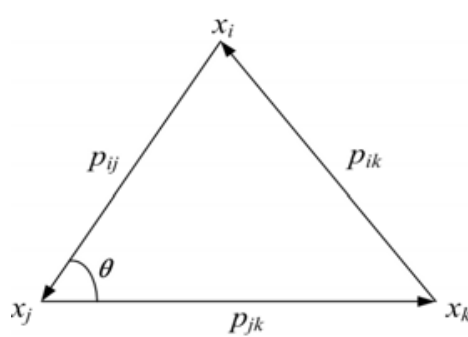

(a)

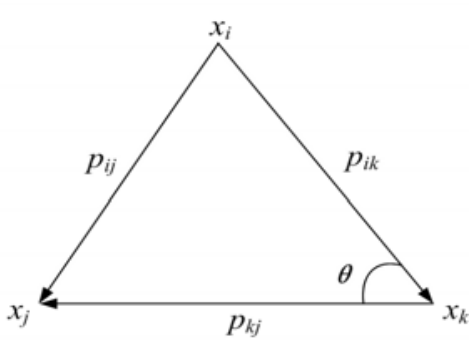

(b)

Fig. 2. Two situations of directed triangle $\triangle x_{i} x_{j} x_{k}$.

\subsubsection{RMMT in the first situation}

In the situation of Fig. $2(a)$, as demonstrated in Section 4.1, given $p_{i j}$ and $p_{j k}$, if the combination 
of $p_{i k}$ with $p_{i j}$ and $p_{j k}$ can form a triangle, then we can determine that $\left|p_{i j}-p_{j k}\right| \leq p_{i k} \leq p_{i j}+p_{j k}$. This condition covers RMMT.

Proposition 3. Let $P=\left(p_{i j}\right)_{n \times n}$ be an FPR matrix on $X \times X$, where $X=\left\{x_{1}, x_{2}, \ldots, x_{n}\right\}$ denotes a set of alternatives. For any three alternatives $x_{i}, x_{j}$, and $x_{k}(i \neq j \neq k)$, when $p_{i j}$ (the degree to which $x_{i}$ is superior to $x_{j}$ ) and $p_{j k}$ (the degree to which $x_{j}$ is superior to $x_{k}$ ) are known, if $p_{i j}, p_{j k}$, and $p_{i k}$ satisfy RMMT, then the three edges with lengths of $p_{i j}, p_{j k}$, and $p_{i k}$ must form a triangle.

Given $p_{i j}$ and $p_{j k}$ such that $p_{i j} \geq 0.5$ and $p_{j k} \geq 0.5$ in Fig. $2(a)$, under the assumption that RMMT is satisfied, Proposition 3 clearly holds because $\left|p_{i j}-p_{j k}\right|<\max \left\{p_{i j}, p_{j k}\right\}$ and $1<p_{i j}+p_{j k}$. In such a triangle composed of $p_{i j}$, $p_{j k}$, and $p_{i k}$, the angle between edge $x_{i} x_{j}$ with length of $p_{i j}$ and edge unu $x_{j} x_{k}$ with length of $p_{j k}$ is determined by

$$
\theta=\arccos \frac{p_{i j}^{2}+p_{j k}^{2}-p_{i k}^{2}}{2 p_{i j} p_{j k}} .
$$

When $p_{i k}$ is limited to $\left[\max \left\{p_{i j}, p_{j k}\right\}, 1\right]$, the range of $\theta$ can be determined.

Property 3. Let $P=\left(p_{i j}\right)_{n \times n}$ be an FPR matrix on $X \times X$, where $X=\left\{x_{1}, x_{2}, \ldots, x_{n}\right\}$ denotes a set of alternatives. For any three alternatives $x_{i}, x_{j}$, and $x_{k}(i \neq j \neq k)$, if their pairwise comparisons $p_{i j}, p_{j k}$, and $p_{i k}$ satisfy $0.5 \leq p_{i j}, p_{j k} \leq 1$ and $\max \left\{p_{i j}, p_{j k}\right\} \leq p_{i k} \leq 1$, then we can determine that the angle between edge ${ }_{x_{i}}^{\text {unu }} x_{j}$ with length $p_{i j}$ and edge $x_{j}^{\text {umu }} x_{k}$ with length $p_{j k}$ in triangle $\Delta x_{i} x_{j} x_{k}$ satisfies

$$
\begin{aligned}
& \arccos \frac{\min \left\{p_{i j}, p_{j k}\right\}}{2 \max \left\{p_{i j}, p_{j k}\right\}} \leq \theta \leq \arccos \frac{p_{i j}^{2}+p_{j k}^{2}-1}{2 p_{i j} p_{j k}}, \\
& \pi / 3 \leq \arccos \frac{\min \left\{p_{i j}, p_{j k}\right\}}{2 \max \left\{p_{i j}, p_{j k}\right\}} \leq 0.4196 \pi, \text { and } \\
& \pi / 3 \leq \arccos \frac{p_{i j}^{2}+p_{j k}^{2}-1}{2 p_{i j} p_{j k}} \leq \pi .
\end{aligned}
$$

The proof of Property 3 is presented in Section A.4 of Appendix A. In order to clearly 
demonstrate Property 3, Example B.1 is given in Section B.3 of Appendix B in the supplementary material. In triangle $\Delta x_{i} x_{j} x_{k}$, where the lengths of edges $\operatorname{umu}_{i} x_{j}$ and $x_{j} x_{k} \quad$ ( $p_{i j}$ and $p_{j k}$, respectively) are provided, and the length of edge $x_{i} x_{k}\left(p_{j k}\right)$ is unknown, when $p_{i j} \geq 0.5$ and $p_{j k} \geq 0.5, p_{i k}$ can be calculated under the assumption that RMMT is satisfied.

Definition 9. Let $P=\left(p_{i j}\right)_{n \times n}$ be an FPR matrix on $X \times X$, where $X=\left\{x_{1}, x_{2}, \ldots, x_{n}\right\}$ denotes a set of alternatives. For any three alternatives $x_{i}, x_{j}$, and $x_{k}(i \neq j \neq k)$, assume that their pairwise comparisons $p_{i j}$ and $p_{j k}$ satisfy $0.5 \leq p_{i j}, p_{j k} \leq 1$ and RMMT is satisfied. Then, in triangle $\triangle x_{i} x_{j} x_{k}$ composed of three edges with lengths of $p_{i j}, p_{j k}$, and $p_{i k}, p_{i k}$ is determined by

$$
p_{i k}=\sqrt{p_{i j}^{2}+p_{j k}^{2}-2 p_{i j} p_{j k} \cos \theta},
$$

where $\theta$ represents the angle between edges $x_{i} x_{j}$ and $x_{j} x_{k}$ in triangle $\Delta x_{i} x_{j} x_{k}$ such that $\arccos \frac{\min \left\{p_{i j}, p_{j k}\right\}}{2 \max \left\{p_{i j}, p_{j k}\right\}} \leq \theta \leq \arccos \frac{p_{i j}^{2}+p_{j k}^{2}-1}{2 p_{i j} p_{j k}}$

\subsubsection{RMMT in the second situation}

In the situation of Fig. $2(b)$, where $p_{i j} \geq 0.5, p_{j k} \leq 0.5$, and $p_{i j} \geq 1-p_{j k}=p_{k j}$, the problem changes to one considering the angle between edges $x_{i} x_{k}$ and $x_{k} x_{j}$ in triangle $\Delta x_{i} x_{j} x_{k}$ under the assumption that RMMT is satisfied. First, the situation where the triangle exists is formally presented.

Proposition 4. Let $P=\left(p_{i j}\right)_{n \times n}$ be an FPR matrix on $X \times X$, where $X=\left\{x_{1}, x_{2}, \ldots, x_{n}\right\}$ denotes a set of alternatives. For any three alternatives $x_{i}, x_{j}$, and $x_{k}(i \neq j \neq k)$, when their pairwise comparisons $p_{i j}, p_{j k}$, and $p_{i k}$ satisfy $p_{i j} \geq 0.5, p_{j k} \leq 0.5$, and $p_{i j} \geq 1-p_{j k}=p_{k j}$, if $p_{i j}, p_{k j}$, and $p_{i k}$ satisfy RMMT and $p_{i j}-p_{k j} \leq p_{i k}$, then three edges with lengths of $p_{i j}, p_{k j}$, and $p_{i k}$ must form a triangle.

Given $p_{i j}$ and $p_{j k}$ such that $p_{i j} \geq 0.5, p_{j k} \leq 0.5$, and $p_{i j} \geq p_{k j}$ in Fig. $2(b)$, under the assumption that RMMT is satisfied and $p_{i j}-p_{k j} \leq p_{i k}$, Proposition 4 clearly holds because $\max \left\{p_{i k}, p_{k j}\right\} \leq p_{i j}$ can be 
used to directly deduce that $p_{i k} \leq p_{i j}+p_{k j}$. In such a triangle, the range of angles between edge $x_{i} x_{k}$ with length $p_{i k}$ and $x_{k} x_{j}$ with length $p_{k j}$ can be determined.

Property 4. Let $P=\left(p_{i j}\right)_{n \times n}$ be an FPR matrix on $X \times X$, where $X=\left\{x_{1}, x_{2}, \ldots, x_{n}\right\}$ denotes a set of alternatives. For any three alternatives $x_{i}, x_{j}$, and $x_{k}(i \neq j \neq k)$, if their pairwise comparisons $p_{i j}, p_{j k}$, and $p_{i k}$ satisfy $p_{i j} \geq 0.5, p_{j k} \leq 0.5, p_{i j} \geq 1-p_{j k}=p_{k j}$, and $0.5 \leq p_{i k} \leq p_{i j}$, then we can determine that the angle between edge $x_{i} x_{k}$ with length $p_{i k}$, and edge $x_{k} x_{j}$ wum with length $p_{k j}$ in triangle $\triangle x_{i} x_{j} x_{k}$ satisfies

$$
\begin{aligned}
& \arccos \frac{\min \left\{p_{i j}, p_{k j}\right\}}{2 \max \left\{p_{i j}, p_{k j}\right\}} \leq \theta \leq \arccos \frac{0.5^{2}+p_{k j}^{2}-p_{i j}^{2}}{2 \cdot 0.5 \cdot p_{k j}} \\
& \pi / 3 \leq \arccos \frac{\min \left\{p_{i j}, p_{k j}\right\}}{2 \max \left\{p_{i j}, p_{k j}\right\}} \leq 0.4196 \pi, \text { and } \\
& \pi / 3 \leq \arccos \frac{0.5^{2}+p_{k j}^{2}-p_{i j}^{2}}{2 \cdot 0.5 \cdot p_{k j}} \leq \pi .
\end{aligned}
$$

The proof of Property 4 is presented in Section A.5 of Appendix A. Example B.2 in Section B.3 clearly demonstrates Property 4. In triangle $\Delta x_{i} x_{j} x_{k}$, where the lengths of edges $\underset{x_{i} x_{j}}{\text { und }} \stackrel{\text { uumu }}{x_{k} x_{j}}$ ( $p_{i j}$ and $p_{k j}$, respectively) are provided and the length of edge $x_{i} x_{k} \quad\left(p_{i k}\right)$ is unknown, when $p_{i j} \geq p_{k j}$ $\geq 0.5, p_{i k}$ can be calculated under the assumption that RMMT is satisfied.

Definition 10. Let $P=\left(p_{i j}\right)_{n \times n}$ be an FPR matrix on $X \times X$, where $X=\left\{x_{1}, x_{2}, \ldots, x_{n}\right\}$ denotes a set of alternatives. For any three alternatives $x_{i}, x_{j}$, and $x_{k}(i \neq j \neq k)$, assume that their pairwise comparisons $p_{i j}$ and $p_{k j}$ satisfy $p_{i j} \geq p_{k j} \geq 0.5$ and RMMT is satisfied. Then, in triangle $\triangle x_{i} x_{j} x_{k}$ composed of three edges with lengths of $p_{i j}, p_{k j}$, and $p_{i k}, p_{i k}$ is determined by

$$
p_{i k}=p_{k j} \cos \theta+\sqrt{p_{i j}^{2}-p_{k j}^{2} \sin ^{2} \theta},
$$

where $\theta$ represents the angle between edges $\quad x_{i} x_{k}$ and $x_{k} x_{j}$ such that $\arccos \frac{\min \left\{p_{i j}, p_{k j}\right\}}{2 \max \left\{p_{i j}, p_{k j}\right\}} \leq$ 
$\theta \leq \arccos \frac{0.5^{2}+p_{k j}^{2}-p_{i j}^{2}}{2 \cdot 0.5 \cdot p_{k j}}$

Eq. (13) is inferred from $p_{i j}=\sqrt{p_{i k}^{2}+p_{k j}^{2}-2 p_{i k} p_{k j} \cos \theta}$ presented in Eq. (9).

\subsection{Introduction of triangular bounded consistency}

By using directed triangles to represent the FPRs for any three alternatives, the RMMT of an FPR matrix is reconstructed in Section 4.2. Taking this as an important foundation, this section introduces a new consistency of an FPR matrix called triangular bounded consistency, which is developed depending on the bounded rationality of a decision maker. Thus, triangular bounded consistency is clearly different from additive and multiplicative consistency. The developed consistency is then applied to estimate the missing elements in an acceptable incomplete FPR matrix.

\subsubsection{Concept of triangular bounded consistency}

As demonstrated in Section 3, when a decision maker provides an FPR matrix, which is additively consistent, they are assumed to be perfectly rational. In contrast, multiplicative consistency relaxes this assumption to guarantee that all FPRs are limited to $[0,1]$. In practice, however, the two types of consistency cannot characterize the bounded rationality of a decision maker. This negatively influences the applicability of the two types of consistency in real cases. To address such a problem, a new consistency of an FPR matrix called triangular bounded consistency is proposed under the condition that RMMT is satisfied.

Triangular bounded consistency is defined under the assumption that a decision maker may not have access to all relevant information or may not be able to process the information consistently and rationally in an efficient way (Yan and Ma, 2015). This guarantees that preferences of the decision maker are reasonably acceptable but not perfectly consistent. The basic idea of checking 
triangular bounded consistency of a known triangle denoted by $\triangle x_{i} x_{j} x_{k}$ as plotted in Fig. $2(a)$ is comprised of four steps:

(1) collecting preferences of a decision maker as directed triangles,

(2) using each directed triangle to determine the third edge $x_{i} x_{k}$ of $\Delta x_{i} x_{j} x_{k}$ from the two edges $x_{i} x_{j}$ and unum $x_{j} x_{k}$

(3) creating a consistency interval based on the mean and standard deviation of the resulting third edges, and

(4) judging whether the known third edge is limited to the created consistency interval to confirm the consistency of the known triangle.

These steps are described in detail below.

\section{(1) Collection of preferences}

There are typically two ways to collect the preferences of a decision maker. One is to choose them from historical evaluations represented by FPRs. The other is to obtain the preferences of the decision maker by asking them to compare given alternatives in pairs in a similar field they are very familiar with. Suppose that the preferences of a decision maker are gathered and expressed by $h$ triangles $\left\{\left(p_{a}^{1}, p_{b}^{1}, p_{c}^{1}\right), \ldots,\left(p_{a}^{h}, p_{b}^{h}, p_{c}^{h}\right)\right\}$. Because the triangles in the second situation presented in Section 4.2 can be transformed into those in the first situation in accordance with the basic characteristics of FPRs in Definition 1 presented in Section 2, the $h$ triangles are assumed in the first situation, where $p_{a}^{m} \geq 0.5, p_{b}^{m} \geq 0.5$, and $p_{c}^{m} \geq \max \left\{p_{a}^{m}, p_{b}^{m}\right\} \quad(m=1, \ldots, h)$ are satisfied. The angle between the two edges associated with $p_{a}^{m}$ and $p_{b}^{m}$ is denoted by $\theta_{m}$, which is limited to $\left[\theta_{m}^{-}, \theta_{m}^{+}\right]=\left[\arccos \frac{\min \left\{p_{a}^{m}, p_{b}^{m}\right\}}{2 \max \left\{p_{a}^{m}, p_{b}^{m}\right\}}, \arccos \frac{\left(p_{a}^{m}\right)^{2}+\left(p_{b}^{m}\right)^{2}-1}{2 p_{a}^{m} p_{b}^{m}}\right]$, as indicated by Property 3 in Section 4.2. 
(2) Determination of the third edge

Given that a known triangle $\Delta x_{i} x_{j} x_{k}$ in the first situation is provided, where the lengths of edges $x_{i} x_{j}$ and $x_{j} x_{k}$ ( $p_{i j}$ and $p_{j k}$, respectively) are larger than or equal to 0.5 , a direct method of using the $h$ triangles to calculate the length of edge $x_{i} x_{k} \quad\left(p_{i k}\right)$ is to regard $\theta_{m}$ as the angle $\theta$ between edges $\begin{aligned} & \text { unum } \\ & x_{i} x_{j}\end{aligned}$ and $x_{j} x_{k}$. We can determine from Eq. (6) that $\theta \in\left[\theta^{-}, \theta^{+}\right]=\left[\arccos \frac{\min \left\{p_{i j}, p_{j k}\right\}}{2 \max \left\{p_{i j}, p_{j k}\right\}}\right.$, $\left.\arccos \frac{p_{i j}^{2}+p_{j k}^{2}-1}{2 p_{i j} p_{j k}}\right]$. For the $h$ triangles, however, $\theta_{m}$ cannot be always guaranteed to be limited to this interval. For example, given $\left(p_{a}^{m}, p_{b}^{m}, p_{c}^{m}\right)=(0.6,0.7,0.85)$ and $\left(p_{i j}, p_{j k}\right)=(0.8,0.8), \theta_{m}=$ $0.4515 \pi$ and $\left[\theta^{-}, \theta^{+}\right]=[0.3333 \pi, 0.4298 \pi]$ can be obtained using Eqs. (5) and (6), respectively. It is clear that $\theta_{m \notin}\left[\theta^{-}, \theta^{+}\right]$, and a direct method is unavailable in this situation.

Therefore, a ratio method is developed to determine $\theta$ in the triangle $\Delta x_{i} x_{j} x_{k}$. The idea of such a method is that the ratio of $\theta_{m}-\theta_{m}^{-}$to $\theta_{m}^{+}-\theta_{m}^{-}$is equal to the ratio of $\theta-\theta^{-}$to $\theta^{+}-\theta^{-}$, as plotted in Fig. 3. Following this idea, we can easily determine that

$$
\theta=\theta^{-}+\frac{\theta_{m}-\theta_{m}^{-}}{\theta_{m}^{+}-\theta_{m}^{-}} \cdot\left(\theta^{+}-\theta^{-}\right)
$$

The value of $p_{i k}$ is then determined using Eq. (9). When all $h$ triangles are applied, $p_{i k}^{m}(m=1, \ldots$, $h)$ is generated.

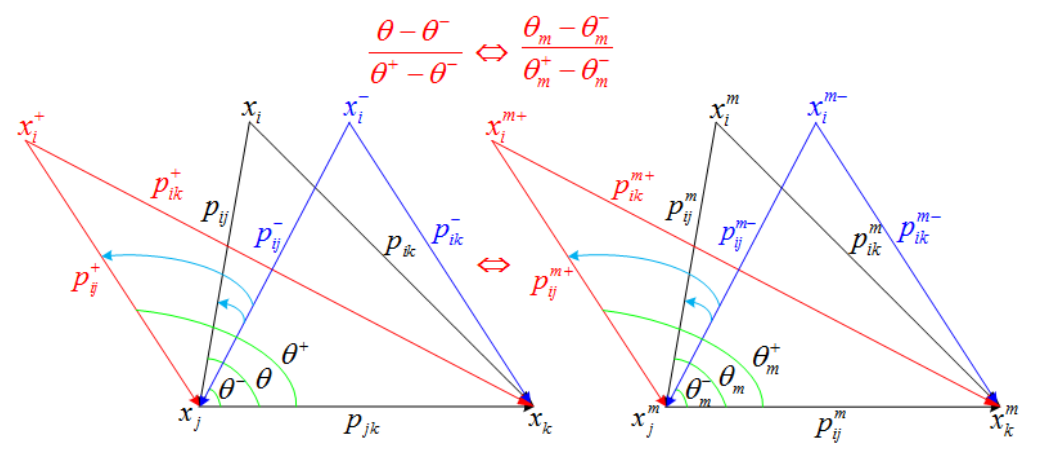

Fig. 3. Equivalent transformation between triangle $\Delta x_{i} x_{j} x_{k}$ and the triangle $\left(p_{a}^{m}, p_{b}^{m}, p_{c}^{m}\right)$

provided by the decision maker. 
It should be noted that $\theta_{m}^{+}=\arccos \frac{\left(p_{a}^{m}\right)^{2}+\left(p_{b}^{m}\right)^{2}-1}{2 p_{a}^{m} p_{b}^{m}}$ and $\theta^{+}=\arccos \frac{p_{i j}^{2}+p_{j k}^{2}-1}{2 p_{i j} p_{j k}}$ are assumed in the above analysis to follow the basic property of FPRs that the maximum value of an FPR is 1 . This property is not ensured by additive consistency; thus, it inspires multiplicative consistency. In theory, the length of the third edge in a triangle may be larger than 1 if the other two edges have lengths larger than 0.5 . From this perspective, additive consistency rather than multiplicative consistency covers all possible situations that may not follow the basic characteristics of FPRs. From the viewpoint of the triangle, $\theta^{+}$should be set to $\pi$ to cover all possible situations. Otherwise, some counterintuitive results may be obtained, which is illustrated by the following example.

Example 2. For a set of alternatives $X=\left\{x_{1}, \ldots, x_{5}\right\}$, suppose that $P=\left(p_{i j}\right)_{5 \times 5}$ is an FPR matrix on $X \times X$ with $p_{12}=0.61, p_{23}=0.75, p_{34}=0.66$, and $p_{45}=0.75$. For simplicity, given $\left(p_{a}^{1}, p_{b}^{1}, p_{c}^{1}\right)=$ $(0.6,0.7,0.78), p_{13}$ and $p_{35}$ are calculated under the condition that $\theta_{1}^{+}=$ $\arccos \frac{\left(p_{a}^{1}\right)^{2}+\left(p_{b}^{1}\right)^{2}-1}{2 p_{a}^{1} p_{b}^{1}}$ and $\theta^{+}=\arccos \frac{p_{i j}^{2}+p_{j k}^{2}-1}{2 p_{i j} p_{j k}}$ as well as $\theta_{1}^{+}=\pi$ and $\theta^{+}=\pi$.

1) $\theta_{1}^{+}=\arccos \frac{\left(p_{a}^{1}\right)^{2}+\left(p_{b}^{1}\right)^{2}-1}{2 p_{a}^{1} p_{b}^{1}}$ and $\theta^{+}=\arccos \frac{p_{i j}^{2}+p_{j k}^{2}-1}{2 p_{i j} p_{j k}}$

In this situation, it can be calculated that $\left[\theta_{1}^{-}, \theta_{1}^{+}\right]=\left[\arccos \frac{\min \left\{p_{a}^{1}, p_{b}^{1}\right\}}{2 \max \left\{p_{a}^{1}, p_{b}^{1}\right\}}\right.$, $\left.\arccos \frac{\left(p_{a}^{1}\right)^{2}+\left(p_{b}^{1}\right)^{2}-1}{2 p_{a}^{1} p_{b}^{1}}\right]=[0.359 \pi, 0.5571 \pi]$, and $\theta_{1}=\arccos \frac{\left(p_{a}^{1}\right)^{2}+\left(p_{b}^{1}\right)^{2}-\left(p_{c}^{1}\right)^{2}}{2 p_{a}^{1} p_{b}^{1}}=0.4071 \pi$. When $p_{12}$ and $p_{23}$ are considered, we can determine that $\left[\theta^{-}, \theta^{+}\right]=\left[\arccos \frac{\min \left\{p_{12}, p_{23}\right\}}{2 \max \left\{p_{12}, p_{23}\right\}}\right.$, $\left.\arccos \frac{p_{12}^{2}+p_{23}^{2}-1}{2 p_{12} p_{23}}\right]=[0.3667 \pi, 0.5228 \pi]$. From $\left[\theta_{1}^{-}, \theta_{1}^{+}\right]$and $\left[\theta^{-}, \theta^{+}\right]$, it can be calculated that $\theta$ $=0.4046 \pi$ using Eq. (14), and it can be further deduced from Eq. (9) that $p_{13}^{1}=0.8151$. As for $p_{34}$ and $p_{45}$, in a similar way, we can determine that $\left[\theta^{-}, \theta^{+}\right]=\left[\arccos \frac{\min \left\{p_{34}, p_{45}\right\}}{2 \max \left\{p_{34}, p_{45}\right\}}\right.$, 
$\left.\arccos \frac{p_{34}^{2}+p_{45}^{2}-1}{2 p_{34} p_{45}}\right]=[0.355 \pi, 0.5006 \pi], \theta=0.3903 \pi$, and $p_{35}^{1}=0.8147$. The conclusion that $p_{13}^{1}>p_{35}^{1}$ is clearly counterintuitive because $p_{12}<p_{34}$ and $p_{23}=p_{45}$.

2) $\theta_{1}^{+}=\pi$ and $\theta^{+}=\pi$

When $\theta_{1}^{+}$and $\theta^{+}$are changed to $\pi$, with respect to $p_{12}$ and $p_{23}, \theta$ is changed to $0.4142 \pi$, in which it can be further deduced that $p_{13}^{1}=0.8313$. Under the same condition, $\theta$ between $p_{34}$ and $p_{35}^{1}$ and between $p_{45}$ and $p_{35}^{1}$ are converted into $0.4034 \pi$ and 0.838 , respectively. The conclusion that $p_{13}^{1}<p_{35}^{1}$ makes sense because it is in line with the given conditions.

Example 2 reveals that $\left[\theta_{m}^{-}, \theta_{m}^{+}\right]=\left[\arccos \frac{\min \left\{p_{a}^{m}, p_{b}^{m}\right\}}{2 \max \left\{p_{a}^{m}, p_{b}^{m}\right\}}, \pi\right]$ for the $h$ triangles and $\left[\theta^{-}, \theta^{+}\right]=$ $\left[\arccos \frac{\min \left\{p_{i j}, p_{j k}\right\}}{2 \max \left\{p_{i j}, p_{j k}\right\}}, \pi\right]$ because the triangle $\Delta x_{i} x_{j} x_{k}$ should be specified to guarantee the rationality of $p_{i k}^{m}$ calculated based on the $h$ triangles. Note that $\theta^{+}=\pi$ may result in $p_{i k}^{m} \geq 1$. As shown in Definition 1 presented in Section 2, $p_{i k}^{m}=1$ means that the alternative $x_{i}$ is absolutely preferred to $x_{k}$. With this consideration, the situation of $p_{i k}^{m}>1$ can be reduced to the situation of $p_{i k}^{m}=1$ without information loss. From this, Property 3 presented in Section 4.2 is also satisfied.

(3) Creation of consistency interval

Each of the $h$ triangles represents the preference of a decision maker and none of them can be considered to be more representative than another without further information provided by the decision maker. In this situation, a feasible method of considering the preferences contained in the $h$ triangles is to find a consistency interval to cover $p_{i k}^{m}(m=1, \ldots, h)$. Because the mean and standard deviation are two statistics commonly used to measure the central tendency and dispersion among a set of discrete numbers, the mean and standard deviation of $p_{i k}^{m} \quad(m=1, \ldots$, h), denoted by 


$$
\begin{aligned}
& \bar{p}_{i k}=\frac{1}{h} \sum_{m=1}^{h} p_{i k}^{m} \text { and } \\
& S_{i k}=\sqrt{\frac{1}{h-1} \sum_{m=1}^{h}\left(p_{i k}^{m}-\bar{p}_{i k}\right)^{2}}
\end{aligned}
$$

respectively, are applied to construct the consistency interval $\left[\bar{p}_{i k}-S_{i k}, \bar{p}_{i k}+S_{i k}\right]$.

(4) Triangular bounded consistency

Using the constructed consistency interval derived from the $h$ triangles provided by a decision maker, whether a triangle $\triangle x_{i} x_{j} x_{k}$ is of triangular bounded consistency is judged as follows.

Definition 11. Suppose that $h$ triangles denoted by $\left\{\left(p_{a}^{1}, p_{b}^{1}, p_{c}^{1}\right), \ldots,\left(p_{a}^{h}, p_{b}^{h}, p_{c}^{h}\right)\right\}$ are provided by a decision maker to characterize their preferences, where $p_{a}^{m} \geq 0.5, \quad p_{b}^{m} \geq 0.5$, and $p_{c}^{m} \geq \max \left\{p_{a}^{m}, p_{b}^{m}\right\}(m=1, \ldots, h)$. Given a triangle $\triangle x_{i} x_{j} x_{k}$ with lengths $p_{i j}, p_{j k}$, and $p_{i k}$ such that $p_{i j}$ $\geq 0.5, p_{j k} \geq 0.5$, and $p_{i k} \geq \max \left\{p_{i j}, p_{j k}\right\}$, assume that $p_{i k}^{m}(m=1, \ldots, h)$ is generated by using Eqs. (9) and (14) and the mean $\bar{p}_{i k}$ and the standard deviation $S_{i k}$ of $p_{i k}^{m}(m=1, \ldots, h)$ are known. Then, $p_{i j}, p_{j k}$, and $p_{i k}$ are considered to satisfy triangular bounded consistency if they satisfy the following condition:

$$
\bar{p}_{i k}-S_{i k} \leq p_{i k} \leq \bar{p}_{i k}+S_{i k}
$$

Based on Definition 11, triangular bounded consistency of an FPR matrix is defined.

Definition 12. Given a set of alternatives $X=\left\{x_{1}, x_{2}, \ldots, x_{n}\right\}$, let $P=\left(p_{i j}\right)_{n \times n}$ be an FPR matrix on $X \times X$. Suppose that $h$ triangles denoted by $\left\{\left(p_{a}^{1}, p_{b}^{1}, p_{c}^{1}\right), \ldots,\left(p_{a}^{h}, p_{b}^{h}, p_{c}^{h}\right)\right\}$ are provided by a decision maker to characterize their preferences, where $p_{a}^{m} \geq 0.5, \quad p_{b}^{m} \geq 0.5$, and $p_{c}^{m} \geq \max \left\{p_{a}^{m}, p_{b}^{m}\right\}(m=1, \ldots, h)$. Under the given conditions, $P$ is said to have triangular bounded consistency if $p_{i j}, p_{j k}$, and $p_{i k}(\forall i, j, k \in\{1, \ldots, n\})$ are verified to be of triangular bounded consistency.

Note that for any three alternatives, we can always find a triangle in the first situation shown in 
Section 4.2, which is similar to the situation of the $h$ triangles and potentially assumed in Definition 12.

\subsubsection{Estimation of missing FPRs}

In real-world applications, it could be a burden on a decision maker to have to compare all alternatives in pairs and guarantee consistency of the comparisons. To relieve this burden, the decision maker is allowed to provide comparisons between some alternatives rather than all alternatives. This gives rise to the question of how to estimate the missing comparisons in an acceptable incomplete comparison matrix as defined in Definition 8 presented in Section 2. This question is addressed using triangular bounded consistency of the matrix for each of the two situations presented in Section 4.2.

(1) Analysis of the first situation

Assume that the $h$ triangles in the first situation, denoted by $\left\{\left(p_{a}^{1}, p_{b}^{1}, p_{c}^{1}\right), \ldots,\left(p_{a}^{h}, p_{b}^{h}\right.\right.$, $\left.\left.p_{c}^{h}\right)\right\}$, are provided by a decision maker. Under the condition that $p_{i j}$ and $p_{j k}$ with $p_{i j} \geq 0.5$ and $p_{j k} \geq$ 0.5 are provided and $p_{i k}$ is unknown, $p_{i k}^{m}(m=1, \ldots, h)$ limited to $[0.5,1], \bar{p}_{i k}$, and $S_{i k}$ can be obtained in accordance with Subsection 4.3.1. When $p_{i k}$ is limited to $\left[\bar{p}_{i k}-S_{i k}, \bar{p}_{i k}+S_{i k}\right], p_{i j}, p_{j k}$, and $p_{i k}$ can be considered to have triangular bounded consistency.

(2) Analysis of the second situation

Suppose that $p_{i j}$ and $p_{k j}$ with $p_{i j} \geq p_{k j} \geq 0.5$ (or $p_{k j} \geq p_{i j} \geq 0.5$ ) are provided; then, the angle between edges $x_{i} x_{k}$ and $x_{k} x_{j}$ in triangle $\Delta x_{i} x_{j} x_{k}$ represented by $\theta$ is limited to $\left[\theta^{-}, \theta^{+}\right]=$ $\left[\arccos \frac{\min \left\{p_{i j}, p_{k j}\right\}}{2 \max \left\{p_{i j}, p_{k j}\right\}}, \pi\right]$. The reason that the upper bound is $\pi$ instead of $\arccos \frac{0.5^{2}+p_{k j}^{2}-p_{i j}^{2}}{2 \cdot 0.5 \cdot p_{k j}}$ is similar to that in the first situation, which is explained in Example 2, so is not discussed here. For each reference triangle $\left(p_{a}^{m}, p_{b}^{m}, p_{c}^{m}\right), \theta$ is also determined from Eq. (14) and is used to 
calculate $p_{i k}^{m}(m=1, \ldots, h)$ in accordance with Eq. (13). Note that $p_{i k}^{m} \geq 0.5$ is specified by Property 4; thus, $p_{i k}^{m}=0.5$ is set when $p_{i k}^{m}<0.5$ because $\theta^{+}=\pi$. Based on $p_{i k}^{m} \quad(m=1, \ldots, h)$ limited to $[0.5,1]$, the consistency interval $\left[\bar{p}_{i k}-S_{i k}, \bar{p}_{i k}+S_{i k}\right]$ is created from Eqs. (15) and (16). Similar to the first situation, any point in the interval can be given to $p_{i k}$ to ensure that $p_{i j}$, $p_{k j}$, and $p_{i k}$ are of triangular bounded consistency.

The above analyses indicate that any value in the consistency interval $\left[\bar{p}_{i k}-S_{i k}, \bar{p}_{i k}+S_{i k}\right]$ can be considered as the estimation of a missing FPR. An important issue is how to find an estimation in which the information contained in $p_{i k}^{m}(m=1, \ldots, h)$ is considered as much as possible. To address this issue, a fair rule is designed to identify such an estimation, which is nearly or even completely equidistant from $p_{i k}^{m}(m=1, \ldots, h)$. In other words, the estimation selected from the consistency interval $\left[\bar{p}_{i k}-S_{i k}, \bar{p}_{i k}+S_{i k}\right]$ is fair for the given $h$ triangles. To find this estimation, an optimization model is constructed as described below.

$$
\begin{array}{ll}
\text { MIN } & \sum_{m=1}^{h}\left(p_{i k}^{*}-p_{i k}^{m}\right)^{2} \\
\text { s.t. } & \bar{p}_{i k}-S_{i k} \leq p_{i k}^{*} \leq \bar{p}_{i k}+S_{i k},
\end{array}
$$

where $p_{i k}^{*}$ symbolizes a decision variable to differentiate it from the optimized $p_{i k}$.

Suppose that $f\left(p_{i k}^{*}\right)=\sum_{m=1}^{h}\left(p_{i k}^{*}-p_{i k}^{m}\right)^{2}$; then, solving the optimization model shown in Eqs. (18) and (19) changes to finding the minimum value of the function $f\left(p_{i k}^{*}\right)$ within the range $\left[\bar{p}_{i k}-S_{i k}, \bar{p}_{i k}+S_{i k}\right]$. For this purpose, the first derivative of $f\left(p_{i k}^{*}\right)$ is calculated as $\frac{d f\left(p_{i k}^{*}\right)}{d p_{i k}^{*}}=$ $2 \sum_{m=1}^{h}\left(p_{i k}^{*}-p_{i k}^{m}\right)$. It can be inferred from $\frac{d f\left(p_{i k}^{*}\right)}{d p_{i k}^{*}}=0$ that $p_{i k}^{*}=\frac{1}{h} \sum_{m=1}^{h} p_{i k}^{m}=\bar{p}_{i k}$ may be an extreme point. To confirm this, the second derivative of $f\left(p_{i k}^{*}\right)$ is obtained as $\frac{d^{2} f\left(p_{i k}^{*}\right)}{d\left(p_{i k}^{*}\right)^{2}}=2 h>0$, 
which indicates that $\frac{d f\left(p_{i k}^{*}\right)}{d p_{i k}^{*}}<0$ when $p_{i k}<\bar{p}_{i k}$ and $\frac{d f\left(p_{i k}^{*}\right)}{d p_{i k}^{*}}>0$ when $p_{i k}>\bar{p}_{i k}$. That is, $f\left(p_{i k}\right)>$ $f\left(p_{i k}^{*}\right)$ with $p_{i k}^{*}=\bar{p}_{i k}$ always holds whether $p_{i k}<\bar{p}_{i k}$ or $p_{i k}>\bar{p}_{i k}$. As a result, it can be concluded that $p_{i k}^{*}=\bar{p}_{i k}$ is the extreme point that minimizes the function $f\left(p_{i k}^{*}\right)$. In other words, $\bar{p}_{i k}$ is the specific estimation selected from the consistency interval $\left[\bar{p}_{i k}-S_{i k}, \bar{p}_{i k}+S_{i k}\right]$ following the fair rule. Such estimation can be used to generate recommended values of missing FPRs in an acceptable incomplete FPR matrix under the condition that Theorem 3 in Section 2 is satisfied.

More importantly, for triangle $\Delta x_{i} x_{j} x_{k}$ with $p_{i j} \geq 0.5$ and $p_{j k}=0.5$, the value of $p_{i k}$ can be estimated for both situations described in Section 4.2. When $p_{j k}=0.5^{+}$, i.e., it is very close to but larger than $0.5, p_{i k}$ can be inferred from the first situation and its value is denoted by $p_{i k}^{+}$. However, when $p_{j k}=0.5^{-}$, i.e., it is very close to but less than $0.5, p_{i k}$ can be obtained from the second situation and its value is denoted by $p_{i k}^{-}$. Without loss of generality, $p_{j k}$ reaching $0.5^{-}$and $p_{j k}$ reaching $0.5^{+}$are equally likely. As a result, the value of $p_{i k}$ can be estimated by the equality $p_{i k}$ $=\left(p_{i k}^{+}+p_{i k}^{-}\right) / 2$.

A numerical example and its discussion are presented in Section B.4 of Appendix B to demonstrate the issue of how to decide whether the known elements in an acceptable incomplete FPR matrix satisfy triangular bounded consistency; the question of how to estimate the missing elements in the matrix by using triangular bounded consistency is also discussed there. It should be noted that when three FPRs provided by a decision maker do not satisfy triangular bounded consistency, they must be revised.

\section{Application of triangular bounded consistency of FPRs in MADA}

In this section, we describe how triangular bounded consistency of FPRs is applied to MADA.

\subsection{Modeling and analysis of MADA problems with FPRs}


An MADA problem arises when a decision maker must compare several alternative courses of action by considering multiple factors to select one or more desirable ones. Pairwise comparison between alternatives is one commonly used approach for MADA owing to its simplicity. Because FPR is one method of recording the decision makers' preferences in pairwise comparisons, in this application, we will apply FPR to analyze the following MADA problem.

Suppose that in an MADA problem $M$ alternatives denoted by $a_{l}(l=1, \ldots, M)$ are compared in pairs on $L$ attributes denoted by $e_{i}(i=1, \ldots, L)$. The comparison between alternatives $a_{l}$ and $a_{m}$ is expressed by FPR to form an FPR matrix on attribute $e_{i}$ denoted by $P\left(e_{i}\left(a_{l m}\right)\right)=\left(p_{i}\left(a_{l m}\right)\right)_{M \times M}$, where $0 \leq p_{i}\left(a_{l m}\right) \leq 1, p_{i}\left(a_{l m}\right)+p_{i}\left(a_{m l}\right)=1$ for $l, m \in\{1, \ldots, M\}$, and $p_{i}\left(a_{l l}\right)=0.5$. The relative weights of the $L$ attributes are denoted by $w=\left(w_{1}, w_{2}, \ldots, w_{L}\right)$, where $0 \leq w_{i} \leq 1$ for $i=1, \ldots, L$ and $\sum_{i=1}^{L} w_{i}=1$ are set to balance the contribution of $p_{i}\left(a_{l m}\right)$ to the overall comparison between alternatives $a_{l}$ and $a_{m}$. The weights can be determined by subjective judgments of a decision maker or can be determined based on the performances of the alternatives (Chin et al., 2015; Fu and Chin, 2014; Fu and $\mathrm{Xu}, 2016)$. Then, the weighted $P\left(e_{i}\left(a_{l m}\right)\right)$ can be combined to generate the overall preferred degree of alternative $a_{l}$ over $a_{m}$, denoted by $p\left(a_{l m}\right)$. The simple additive weighting method (Kaliszewski and Podkopaev, 2016) is one method of carrying out such a combination:

$$
p\left(a_{l m}\right)=\sum_{i=1}^{L} w_{i} \cdot p\left(e_{i}\left(a_{l m}\right)\right)
$$

From the aggregated FPR matrix $P\left(a_{l m}\right)=\left(p\left(a_{l m}\right)\right)_{M \times M}$, desirable alternatives can be identified using a specific method, such as the net flow method (Fodor and Roubens, 1994). In this method, the leaving flow and entering flow of alternative $a_{l}$ are calculated from $\phi^{+}\left(a_{l}\right)=\sum_{m=1, m \neq l}^{M} p\left(a_{l m}\right)$ and $\phi^{-}\left(a_{l}\right)=\sum_{m=1, m \neq l}^{M} p\left(a_{m l}\right)$, respectively. Then, the net flow of alternative $a_{l}$ is calculated by $\phi\left(a_{l}\right)=\phi^{+}\left(a_{l}\right)-\phi^{-}\left(a_{l}\right)$. An alternative with a larger net flow is more preferred; thus, all 
alternatives can be compared by their net flows to generate a ranking order of the $M$ alternatives and desirable ones can be identified. The details of this can be found in Section B.2 of Appendix B.

\subsection{Process of finding solutions to MADA problems with FPRs based on triangular bounded}

\section{consistency}

The above discussion is about finding solutions to MADA problems with complete FPR matrices. This may not be feasible in practice because offering consistent FPR matrices $P\left(a_{l m}\right)_{M \times M}$ is very difficult for a decision maker, particularly when $M$ and $L$ are extremely large. To relieve the burden on the decision maker, the decision maker can offer $(M-1) \cdot L$ FPRs between adjacent alternatives, from which the remaining FPRs can be derived (Sen and Yang, 1994). Following this idea, we design a process of analyzing MADA problems based on triangular bounded consistency, which is plotted in Fig. 4. 


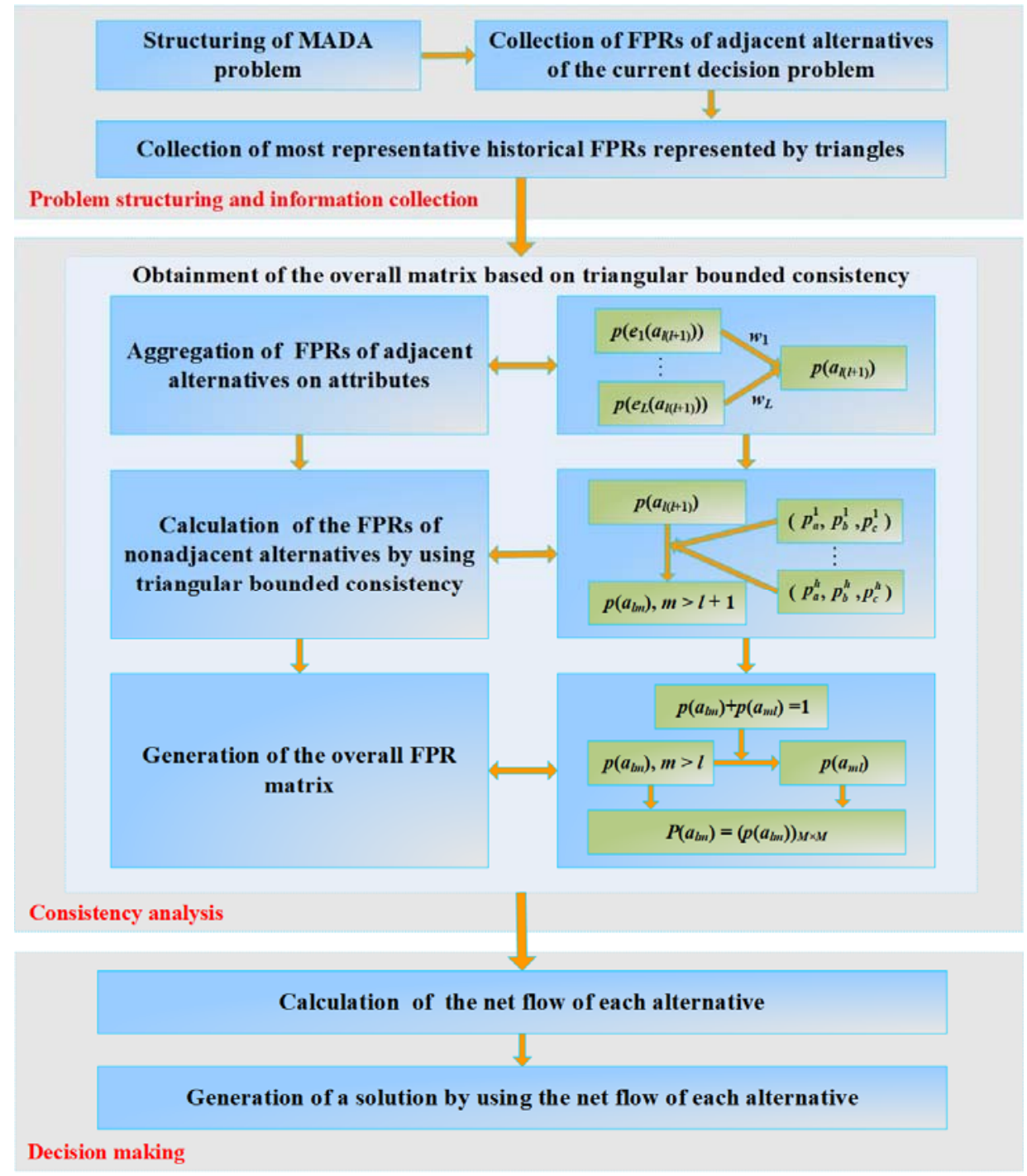

Fig. 4. Process of analyzing MADA problems based on triangular bounded consistency.

Fig. 4 shows the three stages in the process, namely problem structuring and information collection, consistency analysis, and decision-making. In the problem structuring and information collection stage, only $(M-1) \cdot L$ FPRs between adjacent alternatives denoted by $p\left(e_{i}\left(a_{l(l+1)}\right)\right)(l=$ $1, \ldots, M-1)$ are required, which is beneficial for relieving the burden on the decision maker and avoiding inconsistencies in the provided FPRs. This is clearly different from Section 5.1, in which $M \cdot(M-1) \cdot L$ FPRs are required. To estimate FPRs between nonadjacent alternatives, it is necessary to use historical triangles of FPRs obtained from a similar field with which the decision maker is familiar. Suppose that there are $h$ triangles $\left\{\left(p_{a}^{1}, p_{b}^{1}, p_{c}^{1}\right), \ldots,\left(p_{a}^{h}, p_{b}^{h}, p_{c}^{h}\right)\right\}$ available from 
historical FPRs.

In the consistency analysis stage, through Eq. (20), FPRs between adjacent alternatives on each attribute are combined to generate the overall FPR between adjacent alternatives, denoted by $p\left(a_{l(l+1)}\right)(l=1, \ldots, M-1)$. Then, FPRs between nonadjacent alternatives are estimated under the condition that triangular bounded consistency is guaranteed. By considering the $h$ triangles as benchmarks, the FPRs between nonadjacent alternatives $p\left(a_{l m}\right)(\forall l, m \in\{1, \ldots, M\}, m>l+1)$ in the upper triangle of the FPR matrix are derived from $p\left(a_{l(l+1)}\right)$ using triangular bounded consistency, as shown in Definition 11 presented in Subsection 4.3.1 and the optimization model in Eqs. (18) and (19). The FPRs $p\left(a_{m l}\right)(\forall l, m \in\{1, \ldots, M\}, m>l)$ in the lower triangle of the FPR matrix are then obtained in accordance with the basic constraint on FPRs in Definition 1 presented in Section 2, i.e., $p\left(a_{l m}\right)+p\left(a_{m l}\right)=1(\forall l, m \in\{1, \ldots, M\})$.

In the decision-making stage, from the complete FPR matrix, the net flow of each alternative is calculated to generate a solution to the MADA problem.

From the above discussion, it can be observed that the process outlined in Fig. 4 is different from the process of analyzing MADA problems discussed in Section 5.1 in both the problem structuring and information collection stage and the consistency analysis stage. The former involves the estimation of FPRs between nonadjacent alternatives in accordance with triangular bounded consistency. The latter requires complete FPR matrices, which may or may not be consistent.

\section{Case study}

In this section, a problem of selecting areas to market products, i.e., a market selection problem, for a company located in Qingyang County of Anhui Province in China is investigated to 
demonstrate the application of triangular bounded consistency of FPRs in a MADA problem.

\subsection{Description of the market selection problem}

We focus on how a company located in Qingyang County of Anhui province, with limestone powder as their main product, selects markets. In the current situation, the potential markets of the company are the five areas of Anhui, Jiangsu, Shanghai, Belgium, and Japan. Selling limestone powder to these five areas simultaneously imposes a heavy burden on the company because this requires a large amount of operating capital and inventory. To relieve this burden, the company's board decides to select the most appropriate two areas to sell in by considering current cash flow difficulties. The manager of the company acts as the decision maker responsible for the alternative selection with the help of five experts from the sales, planning, finance, and research and development departments and a collaborative university (the second author is the expert from the university). The five alternatives are evaluated in pairs on seven attributes: capital inputs, market demands, process loss, capital cycle, research and development inputs, capabilities of market profits, and capabilities of sustainable development.

Suppose that the five alternatives are denoted by $A_{l}(l=1, \ldots, 5)$ and the seven attributes are denoted by $e_{i}(i=1, \ldots, 7)$. After analyzing the influence of each attribute on the overall comparison between alternatives, the decision maker uses a method discussed by Ölçer and Odabaşi (2005) to specify that $w_{i}(i=1, \ldots, 7)=(0.15,0.25,0.07,0.1,0.1,0.18,0.15)$. Based on the analysis of documents and data related to the five alternatives, the decision maker provides comparisons between adjacent alternatives using MPRs, as presented in Table C.1 of Section C.1 in Appendix C of the supplementary material. The MPRs in Table C.1 are then transformed into FPRs (shown in Table 1) using Proposition B.1. 


\section{Table 1}

FPRs between adjacent alternatives on seven attributes.

\begin{tabular}{ccccc}
\hline Attributes & $p\left(e_{i}\left(A_{12}\right)\right)$ & $p\left(e_{i}\left(A_{23}\right)\right)$ & $p\left(e_{i}\left(A_{34}\right)\right)$ & $p\left(e_{i}\left(A_{45}\right)\right)$ \\
\hline$e_{1}$ & 0.5415 & 0.5766 & 0.6461 & 0.6577 \\
$e_{2}$ & 0.6577 & 0.5217 & 0.6794 & 0.5597 \\
$e_{3}$ & 0.7717 & 0.5000 & 0.6338 & 0.5923 \\
$e_{4}$ & 0.4188 & 0.3838 & 0.4492 & 0.4492 \\
$e_{5}$ & 0.4492 & 0.6070 & 0.6688 & 0.6070 \\
$e_{6}$ & 0.3423 & 0.2915 & 0.3423 & 0.2915 \\
$e_{7}$ & 0.4492 & 0.3423 & 0.4188 & 0.4760 \\
\hline
\end{tabular}

To estimate the FPRs between nonadjacent alternatives, the decision maker is required to choose the triangles of FPRs that he is most confident with from historical evaluations he made for a past decision problem. The problem is to select the most appropriate city to construct a new factory from five candidates: Tongling (TL), Chizhou (CZ), Hefei (HF), Shanghai (SH), and Taizhou (TZ). The five candidates are evaluated on six attributes, which are production cost, manpower resource, regional conditions, service facility, competitor, and political stability. The decision maker is most confident with the FPRs he made when comparing three candidates on each attribute to form the following six triangles: $\{(\mathrm{CZ} / \mathrm{TL}, \mathrm{TL} / \mathrm{SH}, \mathrm{CZ} / \mathrm{SH}),(\mathrm{HF} / \mathrm{SH}, \mathrm{SH} / \mathrm{TL}$, HF/TL), (SH/TZ, TZ/CZ, SH/CZ), (SH/HF, HF/TL, SH/TL), (TZ/CZ, CZ/HF, TZ/HF), (TL/TZ, $\mathrm{TZ} / \mathrm{SH}, \mathrm{TL} / \mathrm{SH})\}=\{(0.59,0.68,0.72),(0.36,0.81,0.70),(0.55,0.76,0.78),(0.64,0.82,0.90)$, $(0.15,0.92,0.83),(0.25,0.29,0.21)\}$. As is stated in Subsection 4.3.1, the triangles in the second situation can be transformed into those in the first situation in accordance with the basic 
characteristics of FPRs in Definition 1 presented in Section 2. For this reason, the above six triangles are uniformly expressed by six ones in the first situation: $\{(0.59,0.68,0.72),(0.64,0.70$, $0.81),(0.55,0.76,0.78),(0.64,0.82,0.90),(0.83,0.85,0.92),(0.75,0.71,0.79)\}$.

\subsection{Generation of a solution to the market selection problem}

To find a solution to the market selection problem, the FPRs between adjacent alternatives $p\left(e_{i}\left(A_{l(l+1)}\right)\right)(i=1, \ldots, 7, l=1, \ldots, 4)$ given in Table 1, which are presented in Section 6.1, are combined using Eq. $(20)$ and $w_{i}(i=1, \ldots, 7)$ to generate the aggregated FPRs between adjacent alternatives $p\left(A_{l(l+1)}\right)(l=1, \ldots, 4)$, as presented in Table 2 .

\section{Table 2}

Aggregated FPRs between the five alternatives.

\begin{tabular}{cccccc}
\hline Areas & $A_{1}$ & $A_{2}$ & $A_{3}$ & $A_{4}$ & $A_{5}$ \\
\hline$A_{1}$ & 0.5 & 0.5155 & 0.497 & 0.561 & 0.6036 \\
$A_{2}$ & 0.4845 & 0.5 & 0.4485 & 0.5039 & 0.557 \\
$A_{3}$ & 0.503 & 0.5515 & 0.5 & 0.5621 & 0.6083 \\
$A_{4}$ & 0.439 & 0.4961 & 0.4379 & 0.5 & 0.5116 \\
$A_{5}$ & 0.3964 & 0.443 & 0.3917 & 0.4884 & 0.5 \\
\hline
\end{tabular}

Then, the overall FPR matrix based on triangular bounded consistency must be obtained, as outlined in the consistency analysis stage of Fig. 4 in Section 5.2. Suppose that the FPRs between nonadjacent alternatives $A_{l}$ and $A_{m}$ in the upper triangle of an FPR matrix are denoted by $p\left(A_{l m}\right)(m$ $>l+1$ ); they must be subsequently estimated. By considering the six triangles in the first situation discussed in Subsection 4.2.1 as benchmarks, six estimations of $p\left(A_{l m}\right)$, denoted by $p^{r}\left(A_{l m}\right) \quad(r=$ $1, \ldots, 6)$, can be obtained. Owing to space limitation, the resulting $p^{r}\left(A_{l m}\right)$ are not provided in this paper. The mean and standard deviation of $p^{r}\left(A_{l m}\right)$, denoted by $\bar{p}\left(A_{l m}\right)$ and $\bar{S}\left(A_{l m}\right)$, 
respectively, can be further calculated using Eqs. (15) and (16) to form the consistency interval of the FPR $p\left(A_{l m}\right)$ and make triangular bounded consistency indicated in Definition 11, which is presented in Subsection 4.3.1. Here, we have $\bar{S}\left(A_{l m}\right) \quad(l=1, \ldots, 3, m>l+1)=(0.003,0.0171$, $0.0175,0.003,0.0171,0.0174)$ for FPRs in the upper triangle of the overall FPR matrix. As presented in Subsection 4.3.2, $\bar{p}\left(A_{l m}\right)$ is the solution to the optimization model given by Eqs. (18) and (19) when the fair rule is adopted; therefore, the aggregated FPRs between nonadjacent alternatives in the upper triangle of the overall FPR matrix are obtained and presented in Table 2. Note that only $\bar{p}\left(A_{l m}\right)$ is presented in Table 2 to avoid repetition. From the FPRs between adjacent and nonadjacent alternatives in the upper triangle of the FPR matrix, all FPRs in the lower triangle are obtained in accordance with the basic constraint on FPRs in Definition 1 presented in Section 2, which can be found in Table 2.

From the results presented in Table 2, the leaving and entering flows of the five alternatives are calculated as $\phi^{+}\left(A_{l}\right)(l=1, \ldots, 5)=(2.1771,1.9939,2.2249,1.8846,1.7195)$ and $\phi^{-}\left(A_{l}\right) \quad(l=$ $1, \ldots, 5)=(1.8229,2.0061,1.7751,2.1154,2.2805)$. Then, $\phi\left(A_{l}\right)$ is derived from $\phi^{+}\left(A_{l}\right)$ and $\phi^{-}\left(A_{l}\right)$ to obtain $(0.3542,-0.0122,0.4498,-0.2308,-0.561)$. A ranking order of the five alternatives is generated from $\phi\left(A_{l}\right)$, which is $A_{3} \mathrm{f} A_{1} \mathrm{f} A_{2} \mathrm{f} A_{4} \mathrm{f} A_{5}$. Based on the ranking order, the most appropriate two areas are Shanghai $\left(A_{3}\right)$ and Anhui $\left(A_{1}\right)$ with the preferential order of $A_{3} \mathrm{f} \quad A_{1}$. Note that the solution is based on the current situation of the company, and it does not mean that the company will not be willing to sell limestone powder to other areas in the future. When the company overcomes its cash flow difficulties, the decision maker may prefer to sell limestone powder to other areas for long-term and sustainable development. 
In the following, we analyze Anhui and Shanghai from real perspectives to demonstrate the above solution. The company has constructed favorable marketing channels in Anhui owing to geographical convenience and thus can satisfy market requirements more rapidly. To sell limestone powder in Anhui, there will be less losses in the transportation process. Owing to the long-term cooperation relationship between the company and consumers in Anhui, the company is capable of achieving a rapid cycle of capital. Despite this, because of the level of economic development in Anhui, the sale market of limestone powder in Anhui is not normative. This negatively contributes to the long-term and sustainable development of the company. Meanwhile, the company has limited capabilities of gaining market profits in Anhui for the same reason.

The situation in Shanghai is different from that in Anhui. For reasons of geographical distance, there will be more loss in the transportation process when the company sells limestone powder to Shanghai. Note that under the given conditions, the company must spend more capital on transportation to guarantee the quality of limestone powder. Owing to the high level of economic development, Shanghai has a normative market and thus provides a good opportunity for the company to gain more market profits. Moreover, Shanghai provides a good business environment, which is beneficial for the capital cycle and the sustainable development of the company. As a whole, the solution above is generated by considering the performances of Anhui and Shanghai on the seven attributes and their weights simultaneously and by conforming to the real situations of Anhui and Shanghai.

\subsection{Influence of historical preferences of the decision maker on solutions}

As shown in Fig. 4, which is presented in Section 5.2, the historical preferences of a decision maker in the problem structuring and information collection stage influence the consistency analysis stage as well as the decision-making stage. They are considered as benchmarks for 
carrying out triangular bounded consistency analysis, and this is reflected in solutions to MADA problems. To examine the potential influence of historical preferences of a decision maker on solutions, we take a given preference represented by a triangle $(0.55,0.76,0.78)$ in the market selection problem as an example to conduct a sensitivity analysis.

To facilitate this examination, we fix the first edge and adapt the second or third edge. First, under the condition that the length of the third edge is changed to 0.9 , suppose that the length of the second edge changes from 0.5 to 0.9 with a step of 0.01 to satisfy RMMT. For the 41 triangles, the net flows of the five alternatives are calculated and plotted in Fig. 5.

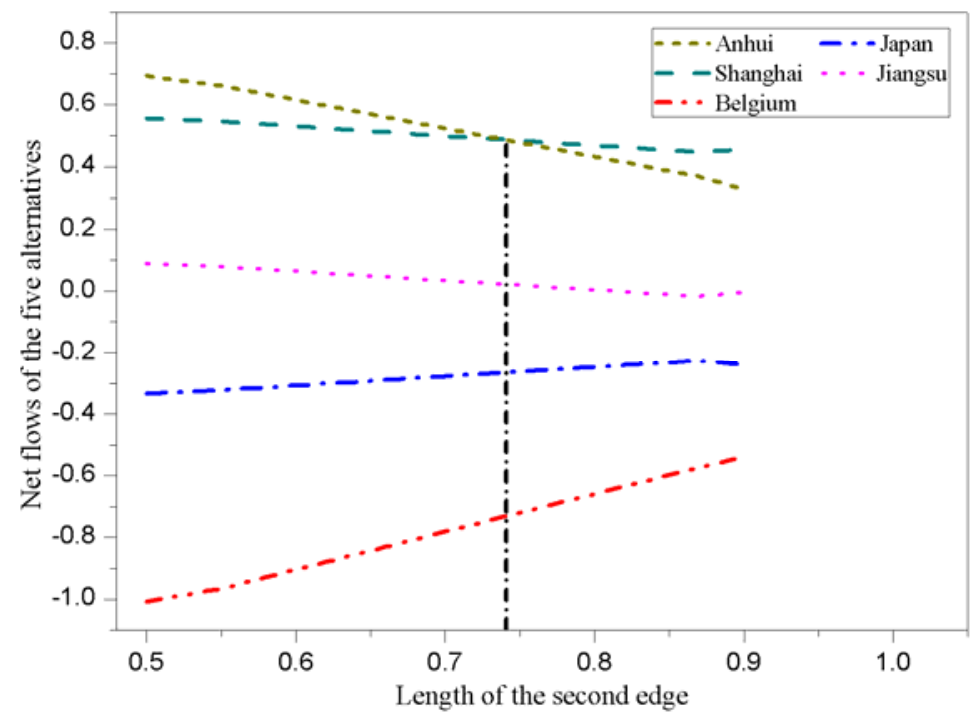

Fig. 5. Movement of net flows of the five alternatives when the length of the second edge changes from 0.5 to 0.9 .

Fig. 5 shows that the rankings of Jiangsu $\left(A_{2}\right)$, Belgium $\left(A_{4}\right)$, and Japan $\left(A_{5}\right)$ remain unchanged with an increase in the length of the second edge, and the rankings of Anhui $\left(A_{1}\right)$ and Shanghai $\left(A_{3}\right)$ alter when the length of the second edge approaches 0.74 . Before and after 0.74, Anhui $\left(A_{1}\right)$ changes from the best area to the second-best area.

Next, when the length of the second edge is set to 0.6 , suppose that the length of the third edge changes from 0.6 to 1 to satisfy RMMT. For the 41 triangles, the net flows of the five alternatives are calculated and plotted in Fig. 6. It is shown in Fig. 6 that the rankings of Jiangsu $\left(A_{2}\right)$, Belgium 
$\left(A_{4}\right)$, and Japan $\left(A_{5}\right)$ remain unchanged with an increase in the length of the third edge, and the rankings of Anhui $\left(A_{1}\right)$ and Shanghai $\left(A_{3}\right)$ alter when the length of the third edge approaches 0.76 .

Before and after 0.76 , Shanghai $\left(A_{3}\right)$ changes from the best area to the second-best area.

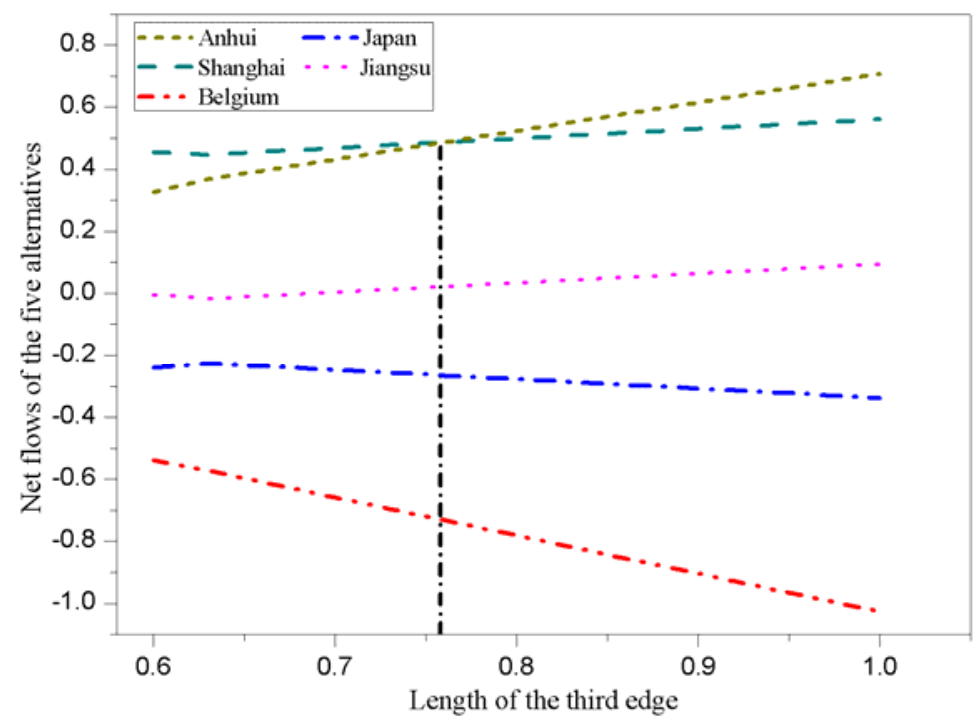

Fig. 6. Movement of net flows of the five alternatives when the length of the third edge changes from 0.6 to 1 .

Altogether, the above discussion illustrates the significant influence of the preferences of the decision maker represented by triangles on solutions to the market selection problem. This also implies that the preferences of a decision maker significantly influence the generation of FPRs between nonadjacent alternatives from FPRs between adjacent alternatives when triangular bounded consistency is followed. Note that perfect rationality can be considered as a special case of bounded rationality. When all preferences of the decision maker satisfy additive or multiplicative consistency of FPRs, triangular bounded consistency will reduce to either additive or multiplicative consistency.

\subsection{Comparative analysis}

As presented in Section 3, triangular bounded consistency is inspired by additive and 
multiplicative consistency. To highlight the difference between triangular bounded consistency and the two types of consistency, we conduct a comparative analysis based on the market selection problem described in Section 6.1.

By using additive and multiplicative consistency indicated in Definitions 2 and 3, respectively, we reimplement the process of generating the aggregated FPRs presented in Section 6.2, and the obtained results are presented in Tables 3 and 4.

\section{Table 3}

Aggregated FPRs between the five alternatives in accordance with additive consistency.

\begin{tabular}{cccccc}
\hline Areas & $A_{1}$ & $A_{2}$ & $A_{3}$ & $A_{4}$ & $A_{5}$ \\
\hline$A_{1}$ & 0.5 & 0.5155 & 0.464 & 0.5261 & 0.5377 \\
$A_{2}$ & 0.4845 & 0.5 & 0.4485 & 0.5106 & 0.5222 \\
$A_{3}$ & 0.536 & 0.5515 & 0.5 & 0.5621 & 0.5737 \\
$A_{4}$ & 0.4739 & 0.4894 & 0.4379 & 0.5 & 0.5116 \\
$A_{5}$ & 0.4623 & 0.4778 & 0.4263 & 0.4884 & 0.5 \\
\hline
\end{tabular}

Table 4

Aggregated FPRs between the five alternatives in accordance with multiplicative consistency.

\begin{tabular}{cccccc}
\hline Areas & $A_{1}$ & $A_{2}$ & $A_{3}$ & $A_{4}$ & $A_{5}$ \\
\hline$A_{1}$ & 0.5 & 0.5155 & 0.4639 & 0.5262 & 0.5378 \\
$A_{2}$ & 0.4845 & 0.5 & 0.4485 & 0.5107 & 0.5223 \\
$A_{3}$ & 0.5361 & 0.5515 & 0.5 & 0.5621 & 0.5735 \\
$A_{4}$ & 0.4738 & 0.4893 & 0.4379 & 0.5 & 0.5116 \\
$A_{5}$ & 0.4622 & 0.4777 & 0.4265 & 0.4884 & 0.5 \\
\hline
\end{tabular}


From the results in Tables 3 and 4 , the net flows of alternative $A_{l}(l=1, \ldots, 5)$ associated with additive and multiplicative consistency denoted by $\phi_{A}\left(A_{l}\right)$ and $\phi_{M}\left(A_{l}\right)$ are calculated as $(0.0866,-0.0684,0.4466,-0.1744,-0.2904)$ and $(0.0868,-0.068,0.4464,-0.1748,-0.2904)$, respectively. A common ranking order of the five alternatives is then generated, which is $A_{3}$ f $A_{1}$ f $A_{2}$ f $A_{4}$ f $A_{5}$. Shanghai $\left(A_{3}\right)$ and Anhui $\left(A_{1}\right)$ are still the most appropriate two areas, which is the same as the solution generated based on triangular bounded consistency. However, the net flow $\phi\left(A_{l}\right)$ is clearly different from $\phi_{A}\left(A_{l}\right)$ and $\phi_{M}\left(A_{l}\right)$, particularly for the net flows of alternatives $A_{1}$ and $A_{5}$. This difference is mainly because the preferences of the decision maker are bounded by triangular bounded consistency rather than additive or multiplicative consistency. Given a fixed set of FPRs of a decision maker, the solutions generated by adopting triangular bounded consistency may not be unique but within a range that reflects the bounded rationality of the decision maker. However, the solution is unique when additive or multiplicative consistency is adopted, implying that all decision makers must be strictly rational and make the same choice no matter who they are. In practice, different decision makers may make different decisions. From this perspective, triangular bounded consistency is more feasible and effective than additive or multiplicative consistency for generating solutions that are acceptable or satisfactory to a decision maker.

In Appendix D of the supplementary material, we further compare the relationship between the two types of consistency and triangular bounded consistency based on the market selection problem.

\section{Conclusions}

Consistency is an important issue concerning FPRs. There are two classical types of consistency 
in existing studies, namely additive and multiplicative consistency. Through a detailed analysis, it is concluded that these two types of consistency cannot reflect the preferences of a decision maker with bounded rationality. To address such an issue, a new consistency measure of an FPR matrix, called triangular bounded consistency, is proposed. Using directed triangles to represent the pairwise FPRs for any three alternatives, the RMMT of FPRs is geometrically represented by the value range of the angles formed by two adjacent edges of triangles. This representation takes different forms in the following two situations. The first is when the length of one of the two adjacent edges is larger than 0.5 , and the length of the other edge is smaller than 0.5 . The second is when the lengths of both the adjacent edges are either larger or smaller than 0.5. Given that RMMT is satisfied, triangular bounded consistency is defined using the preference information of a decision maker characterized by a set of directed triangles. The constructed triangular bounded consistency is then applied to estimate the missing elements in an acceptable incomplete FPR matrix. Finally, a problem of selecting areas to market the products of a company is investigated to demonstrate the application of triangular bounded consistency of FPRs in MADA problems.

In the future, two significant issues concerning triangular bounded consistency must be investigated. The first issue involves the set of triangles provided by a decision maker being filtered to greatly reduce inconsistent triangles that satisfy RMMT but cannot represent the bounded rationality of the decision maker. The other issue involves determining the importance weight of the remaining triangles in determining the lower and upper bounds of triangular bounded consistency of a decision maker. In addition, triangular bounded consistency will be used to compare the consistency levels of different decision makers and check whether a decision maker is more consistent in making some decisions than others. Furthermore, triangular bounded 
consistency will be extended to other situations where preference information of a decision maker is characterized by different types of FPRs, such as triangular FPRs (Meng and Chen, 2017).

\section{References}

A.A. Al Salema, A. Awasthi, Investigating rank reversal in reciprocal fuzzy preference relation based on additive consistency: Causes and solutions, Comput. Ind. Eng. 115 (2018) 573-581.

S.M. Chen, S.H. Cheng, T.E. Lin, Group decision making systems using group recommendations based on interval fuzzy preference relations and consistency matrices, Inf. Sci. 298 (2015) $555-567$.

S.M. Chen, T.E. Lin, L.W. Lee, Group decision making using incomplete fuzzy preference relations based on the additive consistency and the order consistency, Inf. Sci. 259 (2014) 1-15.

F. Chiclana, E. Herrera-Viedma, S. Alonso, F. Herrera, Cardinal consistency of reciprocal preference relations: a characterization of multiplicative transitivity, IEEE Trans. Fuzzy Syst. 17 (1) (2009) 14-23.

K.S. Chin, C. Fu, Y.M. Wang, A method of determining attribute weights in evidential reasoning approach based on incompatibility among attributes, Comput. Ind. Eng. 87 (2015) 150-162.

M. Dasgupta, R. Deb, Transitivity and fuzzy preferences, Soc. Choice Welfare 13 (1996) $305-318$.

X.Y. Deng, Y. Hu, Y. Deng, S. Mahadevan, Supplier selection using AHP methodology extended by D numbers, Expert Syst. Appl. 41 (1) (2014) 156-167.

X.Y. Deng, X. Lu, F.T.S. Chan, R. Sadiq, S. Mahadevan, Y. Deng, D-CFPR: D numbers extended consistent fuzzy preference relations, Knowl.-Based Syst. 73 (2015) 61-68.

J.C. Fodor, M.R. Roubens, Fuzzy Preference Modelling and Multicriteria Decision Support, 
Springer, Netherlands 1994.

C. Fu, K.S. Chin, Robust evidential reasoning approach with unknown attribute weights, Knowl.-Based Syst. 59 (2014) 9-20.

C. Fu, D.L. Xu, Determining attribute weights to improve solution reliability and its application to selecting leading industries, Ann. Oper. Res. 245 (2016) 401-426.

S. Genç, F.E. Boran, D. Akay, Z.S. Xu, Interval multiplicative transitivity for consistency, missing values and priority weights of interval fuzzy preference relations, Inf. Sci. 180 (2010) $4877-4891$.

E. Herowati, U. Ciptomulyono, J. Parunga, Suparno, Expertise-based ranking of experts: An assessment level approach, Fuzzy Sets Syst. 315 (2017) 44-56.

F. Herrera, E. Herrera-Viedma, F. Chiclana, Multiperson decision-making based on multiplicative preference relations, Eur. J. Oper. Res. 129 (1) (2001) 372-385.

E. Herrera-Viedma, F. Herrera, F. Chiclana, M. Luque. Some issues on consistency of fuzzy preference relations. Eur. J. Oper. Res. 154 (2004) 98-109.

I. Kaliszewski, D. Podkopaev, Simple additive weighting-A metamodel for multiple criteria decision analysis methods, Expert Syst. Appl. 54 (2016) 155-161.

J. Krejčí, On multiplicative consistency of interval and fuzzy reciprocal preference relations, Comput. Ind. Eng. 111 (2017) 67-78.

C.C. Li, R.M. Rodríguez, L. Martínez, Y.C. Dong, F. Herrera, Consistency of hesitant fuzzy linguistic preference relations: An interval consistency index, Inf. Sci. 432 (2018) 347-361.

H.C. Liao, Z.S. Xu, Priorities of intuitionistic fuzzy preference relation based on multiplicative consistency, IEEE Trans. Fuzzy Syst. 22 (6) (2014) 1669-1681. 
H.C. Liao, Z.S. Xu, Consistency of the fused intuitionistic fuzzy preference relation ingroup intuitionistic fuzzy analytic hierarchy process, Appl. Soft Comput. 35 (2015) 812-826.

F. Liu, Z.L. Liu, Y.H. Wu, A group decision making model based on triangular fuzzy additive reciprocal matrices with additive approximation-consistency, Appl. Soft Comput. 65 (2018) 349-359.

F. Liu, W.G. Zhang, L.H. Zhang, A group decision making model based on a generalized ordered weighted geometric average operator with interval preference matrices, Fuzzy Sets Syst. 246 (2014) $1-18$.

S. Massanet, J.V. Riera, J. Torrens, E. Herrera-Viedma, A model based on subjective linguistic preference relations for group decision making problems, Inf. Sci. 355-356 (2016) 249-264.

F.Y. Meng, X.H. Chen, A New Method for Triangular Fuzzy Compare Wise Judgment Matrix Process Based on Consistency Analysis, Int. J. Fuzzy Syst. 19 (1) (2017) 27-46.

A.İ. Ölçer, A.Y. Odabaşi, A new fuzzy multiple attributive group decision making methodology and its application to propulsion/manoeuvring system selection problem, Eur. J. Oper. Res. 166 (1) (2005) 93-114.

S.A. Orlovsky, Decision-making with a fuzzy preference relation, Fuzzy Sets Syst. 1 (1978) $155-167$.

W. Pan, K. She, P.Y. Wei, Multi-granulation fuzzy preference relation rough set for ordinal decision system, Fuzzy Sets Syst. 312 (2017) 87-108.

L.G. Pérez, F. Mata, F. Chiclana, G. Kou, E. Herrera-Viedma, Modelling influence in group decision making, Soft Comput. 20 (2016) 1653-1665.

T.L. Saaty, A scaling method for priorities in hierarchy structures, J. Math. Psychol. 15 (1977) 
$234-281$.

P. Sen, J.B. Yang, Design decision making based upon multiple attribute evaluation and minimal preference information, Math. Comput. Model. 20 (3) (1994) 107-124.

H.A. Simon, Models of Bounded Rationality: Empirically Grounded Economic Reason, MIT Press, MA, Cambridge 1982.

T. Tanino, Fuzzy preference orderings in group decision making, Fuzzy Sets Syst. 12 (1984) $117-131$

R. Ureña, F. Chiclana, J.A. Morente-Molinera, E. Herrera-Viedma, Managing incomplete preference relations in decision making: a review and future trends, Inf. Sci. 302 (2015) 14-32.

S.P. Wan, F Wang, J.Y. Dong, Additive consistent interval-valued Atanassov intuitionistic fuzzy preference relation and likelihood comparison algorithm based group decision making, Eur. J. Oper. Res. 263 (2017) 571-582.

S.P. Wan, F. Wang, J.Y. Dong, A group decision making method with interval valued fuzzy preference relations based on the geometric consistency, Inf. Fusion 40 (2018) 87-100.

T.C. Wang, Y.H. Chen, Applying fuzzy linguistic preference relations to the improvement of consistency of fuzzy AHP, Inf. Sci. 178 (2008) 3755-3765.

J. Wu, F. Chiclana, Multiplicative consistency of intuitionistic reciprocal preference relations and its application to missing values estimation and consensus building, Knowl.-Based Syst. 71 (2014) 187-200.

Z.B. Wu, J.P. Xu, Managing consistency and consensus in group decision making with hesitant fuzzy linguistic preference relations, Omega 65 (2016) 28-42.

Y.J. Xu, J.N.D. Gupta, H.M. Wang, The ordinal consistency of an incomplete reciprocal 
preference relation, Fuzzy Sets Syst. 246 (2014) 62-77.

Y.J. Xu, F. Herrera, H.M. Wang, A distance-based framework to deal with ordinal and additive inconsistencies for fuzzy reciprocal preference relations, Inf. Sci. 328 (2016) 189-205.

H.B. Yan, T.J. Ma, A group decision making approach to uncertain quality function deployment based on fuzzy preference relation and fuzzy majority, Eur. J. Oper. Res. 241 (3) (2015) 815-829.

X.X. Zhang, B.F. Ge, J. Jiang, Y.J. Tan, Consensus building in group decision making based on multiplicative consistency with incomplete reciprocal preference relations, Knowl.-Based Syst. 106 (2016) 96-104.

Z. Zhang, X.Y. Kou, W.Y. Yu, C.H. Guo, On priority weights and consistency for incomplete hesitant fuzzy preference relations, Knowl.-Based Syst. 143 (2018) 115-126. 